\title{
Higher-Order Probabilistic Sensitivity Calculations Using the Multicomplex Score Function Method
}

J. Garza and H. Millwater

University of Texas at San Antonio, San Antonio, TX, 78249

\begin{abstract}
The Score Function method used to compute first order probabilistic sensitivities is extended in this work to arbitrary-order derivatives included mixed partial derivatives through the use of multicomplex mathematics. Multicomplex mathematics provides an effective and convenient numerical means to compute the high-order kernel functions with respect to natural parameters or moments (mean and standard deviation) obviating the need to analytically determine the kernel functions. Using these numerical kernel functions, high-order derivatives of the response moments or the probability-of-failure with respect to the parameters of the input distributions can be obtained. Numerical results indicate that the high-order probabilistic sensitivities converge with respect to the number of samples at the same rate as standard Monte Carlo estimates. Implementation of multicomplex mathematics is facilitated through the use of the Cauchy-Riemann matrices; therefore, the extension of common engineering probability distributions to matrix form is presented.
\end{abstract}

Keywords: Score Function; sensitivity analysis; complex Taylor series expansion; multicomplex-step differentiation method

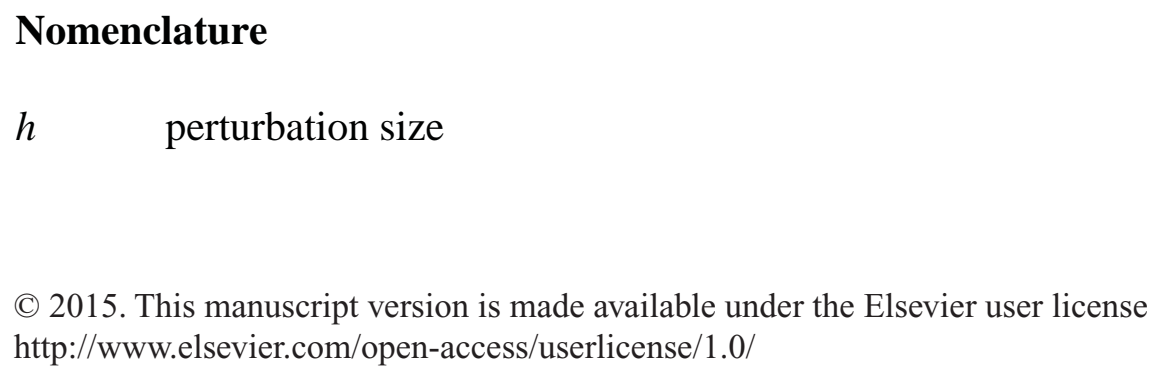




$$
\begin{aligned}
& f \quad \text { scalar function } \\
& \mathrm{N} \quad \text { number of samples } \\
& \mathbf{x} \quad \text { vector of random variables } \\
& z \quad \text { response function } \\
& P_{f} \quad \text { probability-of-failure } \\
& {\left[P_{f}\right] \quad \text { multicomplex probability-of-failure }} \\
& \mu_{Z} \quad \text { response mean } \\
& {\left[\mu_{Z}\right] \quad \text { multicomplex response mean }} \\
& V_{Z} \quad \text { response variance } \\
& {\left[V_{Z}\right] \quad \text { multicomplex response variance }} \\
& \sigma_{Z} \quad \text { response standard deviation } \\
& {\left[\sigma_{Z}\right] \text { multicomplex response standard deviation }} \\
& f_{X}(x) \quad \text { probability density function } \\
& {\left[f_{X}(x)\right] \text { multicomplex probability density function }} \\
& \sigma_{R S} \quad \text { residual strength } \\
& \sigma_{E V D} \quad \text { maximum applied stress } \\
& a_{0} \quad \text { initial crack size } \\
& K_{C} \quad \text { fracture toughness } \\
& Y \quad \text { geometry correction factor } \\
& K_{I} \quad \text { Mode I stress intensity factor } \\
& \text { sqrtM matrix square root } \\
& \log M \quad \text { matrix logarithm }
\end{aligned}
$$


$\exp$ matrix exponential

ГM matrix gamma

$\psi M \quad$ matrix digamma

\section{Introduction}

The Score Function (SF) method is an effective method for computing the sensitivity of a probability estimate with respect to the parameters of the input random variables. That is, one can obtain the partial derivative of an expected value estimate with respect to the parameters of the distribution, e.g., mean, standard deviation, shape, or scale, of the input random variables.

The SF method has some attractive features for sensitivity analysis as outlined below.

\section{Partial derivative based.}

Since SF provides partial derivatives, this information is useful for design modifications. The partial derivatives are computed with respect to the natural parameters or mean and standard deviation of the random variable. Hence, one can project the change in a response with respect to a change in the mean or standard deviation separately. These partial derivatives are global in the sense that the results depend upon the full variation of the random variable. That is, they are local to the random variable parameters but not any particular random variable value. This is analogous to global sensitivity analysis (Saltelli et al., 2008) whose results depend up the parameters of the distribution but not on any particular value of the random variable.

\section{Indifferent to the limit state form.}


$\mathrm{SF}$ is insensitive to the form of the limit state. That is, there is no requirement that the limit state be smooth, differentiable, continuous, etc. Sensitivities for system reliability or component reliability problems can be obtained in an analogous manner.

\section{Inexpensive to compute.}

A significant attraction of SF is that the partial derivatives can be computed for negligle computational cost. The calculation of the partial derivatives is formulated as an expected value with respect to the same joint density function as the original problem. Hence SF uses the same random variable realizations and functional responses that were used during original analysis, e.g., to compute the probability-of-failure or response moments.

\section{Post processing operation.}

As outlined in the previous paragraph, since the existing samples and functional responses are already available, SF merely processes the values within auxilary equations that are simple to compute. Hence it is entirely a post-processing operation. This is beneficial in that SF results can be obtained after the original analysis if the samples and responses are available.

\section{Variance estimates are available.}

The partial derivatives, if computed using random sampling, are themselves random variables. However, high quality variance estimates exist and can be used to construct confidence bounds (Millwater \& Osborn, 2006).

The development of the SF method has progressed steadily over the past few decades. Much of the foundation of the Score Function method has been conducted by Rubinstein and published in a series of papers. The fundamental methodology is 
described in [Rubinstein, 1986; 1989] with applications to discrete event static and dynamic systems. Derivatives up to second order are discussed. Rubinstein and Shapiro (1993) discuss the basic formulation in the context of discrete event systems and demonstrate the use of the Score Function method in stochastic optimization. Wu (1994) applied SF to system reliability analysis and used importance sampling for the probability calculations. Kleijnen and Rubinstein (1996) discuss how SF can be combined with experimental design to reduce the factors to be considered. Rubinstein discusses the optimization of computer models with rare events (1997).

Sues and Cesare (2005) developed the sensitivity of the response mean and standard deviation with respect to the input probability density function (PDF) parameters. Wu and Mohanty (2006) proposed using the methodology as a screening method for problems with a large number of random variables. Millwater and Osborn (2006) applied SF to the probabilistic fatigue analysis of a gas turbine disk and developed variance estimates such that confidence bounds can be computed for each probabilistic sensitivity. Millwater demonstrated that the kernel functions must satisfy certain properties regardless of distribution type. These properties were then used to develop distribution-free analytical expressions of the partial derivatives of the response moments (mean and standard deviation) with respect to the PDF parameters for linear and quadratic response functions (Millwater, 2009). Rahman (2009) provides an application of dimensional reduction and the SF method for calculating stochastic sensitivities. Millwater et al. (2011) extended the kernel functions to the multivariate normal distribution, including derivatives with respect to the correlation coefficient. Millwater and Feng (2011) extended the method to the case of derivatives with respect to the 
bounds of truncated distributions by including a flux term of the PDF across the boundary of the truncated distribution. Lee et al. (2011) use copulas with the SF method to consider correlation between random variables. Millwater et al. (2012) used the SF method to develop a localized sensitivity method that can identify the important region of a probability distribution. Garza and Millwater (2012) applied concepts of the SF to compute the sensitivity of the probability-of-failure with respect to the parameters of a probability-of-detection curve. Wang et al. (2014) developed a methodology using the SF method to compute the derivatives (i.e., first and second-order, including mixed) of the first-order variance contribution of a response with respect to parameters of the random variables.

The extension of SF to high order sensitivities is straightforward in concept but has not been extended to high-order sensitivities primarily due to the complexity of generating high-order kernel functions analytically. Although simple in concept, the calculation of the high-order kernel functions is cumbesome and error prone to compute analytically. A significant complicating factor for high dimensional derivatives is that Jacobian transformations are required to map derivatives with respect to the natural parameters into derivatives with respect to the mean and standard deviation. The Jacobian transformations are needed since most PDFs are defined in terms of natural parameters rather than the mean and standard deviation.

In contrast to the complexities involved using analytical methods, numerical methods using multicomplex mathematics to compute the high-order kernel functions are straightforward to implement, remove the need for Jacobian transformations, and are extendable to arbitrary order derivatives. As a result, the high-order kernel functions are 
computed here numerically using multicomplex mathematics. Since multicomplex numbers can be represented using matrices, the PDFs must be defined in terms of matrices and matrix functions must be used during their evaluation.

\section{Multicomplex Mathematics}

This section introduces the basic concepts of multicomplex mathematics. More details can be found in Price (1991), Lantoine et al. (2012), Millwater and Shirinkam (2014).

\subsection{The Multicomplex Space $\mathbb{C}_{n}$}

The set of all numbers in the $n$th dimensional multicomplex space is denoted by $\mathbb{C}_{n}$. The first case, $n=0$, is defined as the set of all real numbers (i.e., $\mathbb{C}_{0}:=\mathbb{R}$ ). The second case, $n=1$, is defined as the set of all complex numbers (i.e., $\mathbb{C}_{1}:=\mathbb{C}$ ). These two number systems should be very familiar, in that the rules of algebraic operation for numbers and functions defined on these spaces are well known.

Multicomplex numbers are a multi-dimensional generalization of complex numbers. Like complex numbers, multicomplex addition and multiplication are associative and commutative. The first of an infinite number of multicomplex spaces is the bicomplex space. The set of all bicomplex numbers is denoted by $\mathbb{C}_{2}$. Next, $\mathbb{C}_{3}$ denotes the set of all tricomplex numbers, $\mathbb{C}_{4}$ denotes the set of all quadcomplex numbers, etc.

There are various ways to represent a multicomplex number in $\mathbb{C}_{n}$ through isomorphisms. Two mathematical systems are isomorphic if there exists a one-to-one 
correspondence between the systems. As shown in the next section, there is a matrix algebra, which is isomorphic to $\mathbb{C}_{n}$.

\subsection{Multicomplex Step Differentiation Method}

The multicomplex step differentiation (MCX) method can be used to calculate higher-order derivatives of a function of several variables and parameters (Lantoine et al., 2012), as long as the function is holomorphic in the variable(s) or parameter(s) of interest. For example, a second-order derivative of a function $f(x, y)$ with respect to $x$ can be computed by perturbing $x$ in two imaginary unit directions (i.e., $x+h\left(i_{1}+i_{2}\right)$ ) prior to evaluating the function, where $h$ is a numerically small number, e.g., $h=10^{-10}$. As a byproduct a first-order derivative is also calculated. The function and its derivatives are obtained as follows:

$$
\begin{aligned}
& f(x, y) \approx \operatorname{Re}\left[f\left(x+h\left(i_{1}+i_{2}\right), y\right)\right] \\
& \frac{\partial f(x, y)}{\partial x} \approx \frac{\operatorname{Im}_{1}\left[f\left(x+h\left(i_{1}+i_{2}\right), y\right)\right]}{h}=\frac{\operatorname{Im}_{2}\left[f\left(x+h\left(i_{1}+i_{2}\right), y\right)\right]}{h} \\
& \frac{\partial^{2} f(x, y)}{\partial x^{2}} \approx \frac{\operatorname{Im}_{12}\left[f\left(x+h\left(i_{1}+i_{2}\right), y\right)\right]}{h^{2}}
\end{aligned}
$$

where $\operatorname{Re}[\cdot], \operatorname{Im}_{1}[\cdot], \operatorname{Im}_{2}[\cdot]$, and $\operatorname{Im}_{12}[\cdot]$ denote the real part, $i_{1}$ imaginary part, $i_{2}$ imaginary part and $i_{1} i_{2}$ imaginary part, respectively. The properties for the imaginary unit directions are $i_{1}^{2}=i_{2}^{2}=-1$ and $i_{1} i_{2}=i_{2} i_{1}$. Similarly, derivatives with respect $y$ to can be obtained by evaluating $f\left(x, y+h\left(i_{1}+i_{2}\right)\right)$. A mixed second-order derivative can be computed by evaluating $f\left(x+h i_{1}, y+h i_{2}\right)$ or $f\left(x+h i_{2}, y+h i_{1}\right)$.

A matrix representation can be used to address the computation of multicomplex numbers. Inserting the matrices in the function of interest, $f$, results in a matrix output 
$[f]$, where the brackets denote a matrix. The dimensions of $[f]$ are the dimensions of the input matrices, e.g., $2 \times 2$ for complex, 4 x 4 for bicomplex, $8 \times 8$ for tricomplex, $16 \times$ 16 for quadcomplex, etc. For example, in order to compute the mixed derivative, a perturbation in both $x$ and $y$ is required; hence each variable is perturbed along a separate imaginary unit direction. In this case, the bicomplex matrices are

$$
\begin{aligned}
& X=x+i_{1} h+i_{2} 0+i_{1} i_{2} 0 \\
& \leftrightarrow[X]=\left[\begin{array}{cccc}
x & -h & 0 & 0 \\
h & x & 0 & 0 \\
0 & 0 & x & -h \\
0 & 0 & h & x
\end{array}\right] \\
& Y=y+i_{1} 0+i_{2} h+i_{1} i_{2} 0 \\
& \leftrightarrow[Y]=\left[\begin{array}{cccc}
y & 0 & -h & 0 \\
0 & y & 0 & -h \\
h & 0 & y & 0 \\
0 & h & 0 & y
\end{array}\right]
\end{aligned}
$$

The output of the matrix-valued function is

$$
[f([X],[Y])]=\left[\begin{array}{cccc}
f(x, y) & -h \frac{\partial f(x, y)}{\partial x} & -h \frac{\partial f(x, y)}{\partial y} & h^{2} \frac{\partial^{2} f(x, y)}{\partial x \partial y} \\
h \frac{\partial f(x, y)}{\partial x} & f(x, y) & -h^{2} \frac{\partial^{2} f(x, y)}{\partial x \partial y} & -h \frac{\partial f(x, y)}{\partial y} \\
h \frac{\partial f(x, y)}{\partial y} & -h^{2} \frac{\partial^{2} f(x, y)}{\partial x \partial y} & f(x, y) & -h \frac{\partial f(x, y)}{\partial x} \\
h^{2} \frac{\partial^{2} f(x, y)}{\partial x \partial y} & h \frac{\partial f(x, y)}{\partial y} & h \frac{\partial f(x, y)}{\partial x} & f(x, y)
\end{array}\right]
$$

As shown in Eq. (6), the function $f$ and its derivatives are solely contained in column one of the resulting matrix-valued function. Equation (6) is isomorphic to 
$f(x, y)+h \frac{\partial f(x, y)}{\partial x} i_{1}+h \frac{\partial f(x, y)}{\partial y} i_{2}+h^{2} \frac{\partial^{2} f(x, y)}{\partial x \partial y} i_{1} i_{2}$. The function and its derivatives with

respect to $x$ and $y$ are given by the following formulas:

$$
\begin{aligned}
& f(x, y) \approx[f([X],[Y])]_{1,1} \\
& \frac{\partial f(x, y)}{\partial x} \approx \frac{[f([X],[Y])]_{2,1}}{h} \\
& \frac{\partial f(x, y)}{\partial y} \approx \frac{[f([X],[Y])]_{3,1}}{h} \\
& \frac{\partial^{2} f(x, y)}{\partial x \partial y} \approx \frac{[f([X],[Y])]_{4,1}}{h^{2}}
\end{aligned}
$$

where the subscript $i, j$ indicates row $i$ and column $j$ of the indicated matrix.

As mentioned by Lantoine (2012), there are practical limits on the step size values that can be chosen when using finite precision arithmetic. That is, when using double precision numbers, the upper bound of the step size value is $h=10^{-8}$. This is because the truncation error for an arbitrary order multicomplex step derivative is on the order of $h^{2}$ (i.e., $h^{2}<\varepsilon \approx 10^{-16}$ ). Furthermore, $10^{-308}$ is the smallest nonzero number that can be represented in double precision arithmetic. Since $h^{n}$ appears in the highest order derivative to be computed, one must ensure that $h^{n}>10^{-308}$. Thus, for a multicomplex analysis of order $n$, the bounds on $h$ are $10^{-308 / n}<h<10^{-8}$ when using double precision.

\section{The Score Function Method}

A brief overview of the SF method as applied to independent random variables and correlated normal variables is presented then extended to multicomplex mathematics. A more detailed discussion of the SF method can be found in Rubinstein et al. (1993), 
Millwater (2009; 2011), Garza and Millwater (2012), Arnst and Ponthot (2014), and Wang et al. (2014).

An important metric in reliability analysis is the probability-of-failure (POF), $P_{f}$. The POF is the probability that a system response function will exceed or fall short of a specified threshold level. This threshold level can be seen as a design requirement, and is typically expressed in terms of a limit state function, also called a performance function. The limit state function is denoted by $g(\mathbf{x})$ with $\mathbf{x}$ being a vector of random variables, $\mathbf{x}=\left\{x_{1}, x_{2}, \ldots, x_{m}\right\}$

The POF is computed as

$$
P_{f}=\int_{\mathbf{g}(\mathbf{x}) \leq \mathbf{0}} f_{X}(\mathbf{x} ; \boldsymbol{\theta}) d \mathbf{x}
$$

where the $m$-dimensional integral corresponds to the $m$ number of random variables, $f_{\mathbf{X}}(\mathbf{x} ; \boldsymbol{\theta})$ is the joint probability density function (JPDF), $\boldsymbol{\theta}$ is a vector of density function parameters, and $g(\mathbf{x}) \leq 0$ is used to define the failure region.

Generally, in the cases of higher dimensions, the limits of integration pertaining to the failure region are not known, so the POF is written in terms of the indicator function $I(\mathbf{x})$.

$$
\begin{aligned}
P_{f} & =\int_{-\infty}^{\infty} I(\mathbf{x}) f_{\mathbf{X}}(\mathbf{x} ; \boldsymbol{\theta}) d \mathbf{x} \\
& =E[I(\mathbf{x})]
\end{aligned}
$$

where $E[\cdot]$ is the expected value operator. The indicator function equals one when $g(\mathbf{x}) \leq 0$ and zero otherwise. 


$$
I(\mathbf{x})=\left\{\begin{array}{lll}
0 & g(\mathbf{x})>0 & \text { safe } \\
1 & g(\mathbf{x}) \leq 0 & \text { fail }
\end{array}\right.
$$

The $n$th order derivative of the POF with respect to a parameter of the random variable $i$, denoted as $\theta_{i}$, can be written as

$$
\begin{aligned}
\frac{\partial^{n} P_{f}}{\partial \theta_{i}^{n}} & =\int_{-\infty}^{\infty} I(\mathbf{x}) \cdot \frac{\partial^{n} f_{\mathbf{X}}(\mathbf{x} ; \boldsymbol{\theta})}{\partial \theta_{i}^{n}} d \mathbf{x} \\
& =\int_{-\infty}^{\infty} I(\mathbf{x}) \cdot \frac{\partial^{n} f_{\mathbf{X}}(\mathbf{x} ; \boldsymbol{\theta})}{\partial \theta_{i}^{n}} \frac{f_{\mathbf{X}}(\mathbf{x} ; \boldsymbol{\theta})}{f_{\mathbf{X}}(\mathbf{x} ; \boldsymbol{\theta})} d \mathbf{x} \\
& =\int_{-\infty}^{\infty} I(\mathbf{x}) \cdot\left(\frac{\partial^{n} f_{\mathbf{X}}(\mathbf{x} ; \boldsymbol{\theta})}{\partial \theta_{i}^{n}} \frac{1}{f_{\mathbf{X}}(\mathbf{x} ; \boldsymbol{\theta})}\right) f_{\mathbf{X}}(\mathbf{x} ; \boldsymbol{\theta}) d \mathbf{x} \\
& =\int_{-\infty}^{\infty} I(\mathbf{x}) \cdot \kappa_{\theta_{i}}^{(n)}(\mathbf{x}) f_{\mathbf{X}}(\mathbf{x} ; \boldsymbol{\theta}) d \mathbf{x} \\
& =E\left[I(\mathbf{x}) \cdot \kappa_{\theta_{i}}^{(n)}(\mathbf{x})\right]
\end{aligned}
$$

where $\kappa$ is called the kernel function. For independent random variables, the standard form of the $n$th order kernel function is defined as

$$
\kappa_{\theta_{i}}^{(n)}(x)=\frac{\partial^{n} f_{X}(x)}{\partial \theta_{i}^{n}} \frac{1}{f_{X}(x)}
$$

For $n=1, \kappa_{\theta_{i}}^{(1)}(x)=\frac{\partial \ln \left(f_{X}(x)\right.}{\partial \theta_{i}}=\frac{\partial f_{X}(x)}{\partial \theta_{i}} \frac{1}{f_{X}(x)}$ which is the definition of the score function SF from statistics, hence the name of the method.

Mixed partial derivatives can be determined as

$$
\begin{gathered}
\frac{\partial^{n 1+n 2} P_{f}}{\partial \theta_{i}^{n 1} \partial \theta_{j}^{n 2}}=\int_{-\infty}^{\infty} I(\mathbf{x}) \cdot \frac{\partial^{n 1+n 2} f_{\mathbf{X}}(\mathbf{x} ; \boldsymbol{\theta})}{\partial \theta_{i}^{n 1} \partial \theta_{j}^{n 2}} d \mathbf{x} \\
=E\left[I(\mathbf{x}) \cdot \kappa_{\theta_{i} \theta_{j}}^{(n 1, n 2)}(\mathbf{x})\right]
\end{gathered}
$$

where $\theta_{i}$ and $\theta_{j}$ are parameters from random variables $X_{i}$ and $X_{j}$.

Similarly, the $n$th order derivative of the mean response of a system with respect to $\theta_{i}$ is expressed as 


$$
\begin{aligned}
\frac{\partial^{n} \mu_{Z}}{\partial \theta_{i}^{n}} & =\int_{-\infty}^{\infty} z(\mathbf{x}) \cdot \kappa_{\theta_{i}}^{(n)}\left(x_{i}\right) \cdot f_{\mathbf{X}}(\mathbf{x} ; \boldsymbol{\theta}) d \mathbf{x} \\
& =E\left[z(\mathbf{x}) \cdot \kappa_{\theta_{i}}^{(n)}\left(x_{i}\right)\right]
\end{aligned}
$$

where $z(\mathbf{x})$ is a system response function.

The analytical expressions for the response variance and standard deviation derivatives with respect to $\theta_{i}$ require the application of the product rule and chain rule.

For instance, the first-order derivative of the response variance is

$$
\begin{aligned}
\frac{\partial V_{Z}}{\partial \theta_{i}} & =\int_{-\infty}^{\infty} z(\mathbf{x})^{2} \cdot \kappa_{\theta_{i}}^{(1)}\left(x_{i}\right) \cdot f_{\mathbf{X}}(\mathbf{x} ; \boldsymbol{\theta}) d \mathbf{x}-2 \mu_{Z} \frac{\partial \mu_{Z}}{\partial \theta_{i}} \\
& =E\left[z(\mathbf{x})^{2} \cdot \kappa_{\theta_{i}}^{(1)}\left(x_{i}\right)\right]-2 \mu_{Z} \cdot E\left[z(\mathbf{x}) \cdot \kappa_{\theta_{i}}^{(1)}\left(x_{i}\right)\right]
\end{aligned}
$$

Using the relationship $\sigma_{Z}^{2}=V_{Z}$, the first-order derivative of the response standard deviation is

$$
\begin{aligned}
\frac{\partial \sigma_{Z}}{\partial \theta_{i}} & =\frac{\partial V_{Z}}{\partial \theta_{i}} \frac{1}{2 \sigma_{Z}} \\
& =\frac{E\left[z(\mathbf{x})^{2} \cdot \kappa_{\theta_{i}}^{(1)}\left(x_{i}\right)\right]-2 \mu_{Z} \cdot E\left[z(\mathbf{x}) \cdot \kappa_{\theta_{i}}^{(1)}\left(x_{i}\right)\right]}{2 \sigma_{Z}}
\end{aligned}
$$

Higher-order partial derivatives of the variance and standard deviation are obtained by repeatedly differentiating Eqs. $(18,19)$. However, as the derivative order increases, the complexity of the solution also increases. For example, the exact fourthorder derivative of the response standard deviation with respect to parameters $\theta_{i} \theta_{j} \theta_{k} \theta_{l}$ is expressed as 


$$
\begin{aligned}
\frac{\partial^{4} \sigma_{Z}}{\partial \theta_{i} \partial \theta_{j} \partial \theta_{k} \partial \theta_{l}}= & \frac{1}{2}\left[\frac{1}{\sigma_{Z}} V_{Z, i j k l}-\frac{1}{\sigma_{Z}^{2}} \sigma_{Z, l} V_{Z, i j k}+\frac{2}{\sigma_{Z}^{3}} \sigma_{Z, l} \sigma_{Z, k} V_{Z, i j}-\frac{1}{\sigma_{Z}^{2}} \sigma_{Z, k l} V_{Z, i j}-\frac{1}{\sigma_{Z}^{2}} \sigma_{Z, k} V_{Z, i j l}\right. \\
& -\frac{6}{\sigma_{Z}^{4}} \sigma_{Z, l} \sigma_{Z, k} \sigma_{Z, j} V_{Z, i}+\frac{2}{\sigma_{Z}^{3}} \sigma_{Z, k l} \sigma_{Z, j} V_{Z, i}+\frac{2}{\sigma_{Z}^{3}} \sigma_{Z, k} \sigma_{Z, j l} V_{Z, i}+\frac{2}{\sigma_{Z}^{3}} \sigma_{Z, k} \sigma_{Z, j} V_{Z, i l}+ \\
& \frac{2}{\sigma_{Z}^{3}} \sigma_{Z, l} \sigma_{Z, j k} V_{Z, i}-\frac{1}{\sigma_{Z}^{2}} \sigma_{Z, j k l} V_{Z, i}-\frac{1}{\sigma_{Z}^{2}} \sigma_{Z, j k} V_{Z, i l}+\frac{2}{\sigma_{Z}^{3}} \sigma_{Z, l} \sigma_{Z, j} V_{Z, i k}-\frac{1}{\sigma_{Z}^{2}} \sigma_{Z, j l} V_{Z, i k} \\
& \left.-\frac{1}{\sigma_{Z}^{2}} \sigma_{Z, j} V_{Z, k l}\right]
\end{aligned}
$$

where $\theta_{i}, \theta_{j}, \theta_{k}$, and $\theta_{l}$ are parameters of the $i$ th, $j$ th, $k$ th, and $l$ th random variable respectively. In Eq. (20), the derivatives on the right-hand side of the equal sign are written in compact form by using indicial notation, e.g., $V_{Z, i j k l}=\partial^{4} V_{Z} / \partial \theta_{i} \partial \theta_{j} \partial \theta_{k} \partial \theta_{l}$. The multicomplex SF method obviates the need for these analytical derivatives, clearly highlighting the advantage of the method.

The expressions given in Eqs. (14,16-19) require the evaluation of the kernel

functions. Analytically deriving high order kernel functions $\kappa_{\theta_{i}}^{(n)}(x)=\frac{\partial^{n} f_{X}(x)}{\partial \theta_{i}^{n}} \frac{1}{f_{X}(x)}$ is at best cumbersome and error prone. To facilitate this process, the MCX method can be used to obtain the POF and response moment derivatives with ease. The disadvantage is that the formal definitions used to define PDFs must be extended to accommodate matrix forms for its parameters.

\subsection{Higher-Order Kernel Functions Using the MCX Method}

The MCX-SF method computes arbitrary order derivatives of a statistical estimate with respect to parameters of a PDF through multicomplex mathematics. Identical to the traditional SF method, the MCX-SF method can be integrated with any sampling method, 
e.g., standard Monte Carlo sampling, Latin hypercube, importance sampling, etc. or other numerical integration methods.

The higher order sensitivities, including mixed terms, are calculated numerically using suitable modifications of the SF method as described below. Most importantly, there is no need to analytically differentiate the random variable PDFs to develop the requisite kernel functions. In the MCX-SF method, the differentiation is performed numerically using the matrix representation of a multicomplex number. The main requirement is that the PDFs must be holomorphic with respect to the parameter(s) of interest.

A first-order kernel function approximation is presented using complex numbers to facilitate understanding. Subsequently, multicomplex numbers are used to derive higher-order kernel functions.

The first-order kernel function of the parameter $\theta_{i}$ can be approximated as

$$
\kappa_{\theta_{i}}^{(1)}(x)=\frac{\partial \ln \left(f_{X}(x)\right)}{\partial \theta_{i}} \approx \frac{\operatorname{Im}\left[\ln \left(f\left(x, \theta_{i}+i h\right)\right)\right]}{h}
$$

As an example, let $\mu$ represent the mean parameter of a normal distribution. The quasianalytic first-order kernel function approximation using the complex-step differentiation method can be derived as (see Appendix A)

$$
\kappa_{\mu}(x)=\frac{d \ln \left(f_{X}(x)\right)}{d \mu} \approx \frac{\operatorname{Im}\left[\ln \left(f_{X}(x, \mu+i h)\right)\right]}{h}=\frac{(x-\mu)}{\sigma^{2}}
$$

This quasi-analytic solution is in agreement with the analytical solution. In practice, the computation of the kernel function is performed numerically.

For higher-order partial derivatives of the statistical estimates with respect to $\theta_{i}$, a multicomplex perturbation must be added to the parameter $\theta_{i}$ accordingly. The 
multicomplex perturbation depends on the order of the derivative to be computed. Let $\left[\theta_{i}\right]$ represent the matrix form of the corresponding perturbed parameter. The multicomplex-valued (matrix form) POF and response moments are shown below.

$$
\begin{aligned}
& {\left[P_{f}\left(\left[\theta_{i}\right]\right)\right] \approx \frac{1}{N} \sum_{k=1}^{N} I\left(\mathbf{x}_{k}\right) \cdot \frac{\left[f_{X_{i}}\left(x_{i, k} ;\left[\theta_{i}\right]\right)\right]}{f_{X_{i}}\left(x_{i, k}\right)}} \\
& {\left[\mu_{Z}\left(\left[\theta_{i}\right]\right)\right] \approx \frac{1}{N} \sum_{k=1}^{N} z\left(\mathbf{x}_{k}\right) \cdot \frac{\left[f_{X_{i}}\left(x_{i, k} ;\left[\theta_{i}\right]\right)\right]}{f_{X_{i}}\left(x_{i, k}\right)}} \\
& {\left[V_{Z}\left(\left[\theta_{i}\right]\right)\right] \approx \frac{1}{N} \sum_{k=1}^{N} z\left(\mathbf{x}_{k}\right)^{2} \cdot \frac{\left[f_{X_{i}}\left(x_{i, k} ;\left[\theta_{i}\right]\right)\right]}{f_{X_{i}}\left(x_{i, k}\right)}-\left[\mu_{Z}\left(\left[\theta_{i}\right]\right)\right]^{2}}
\end{aligned}
$$

where $f_{X_{i}}\left(x_{i, k}\right)=\left[f_{X_{i}}\left(x_{i, k} ;\left[\theta_{i}\right]\right)\right]_{1,1}$ is the PDF of the $i$ th random variable and $\left[f_{X_{i}}\left(x_{i, k} ;\left[\theta_{i}\right]\right)\right]_{1,1}$ is the first element in the matrix-valued PDF. The corresponding multicomplex standard deviation is calculated as

$$
\left[\sigma_{Z}\left(\left[\theta_{i}\right]\right)\right]=\operatorname{sqrtM}\left(\left[V_{Z}\left(\left[\theta_{i}\right]\right)\right]\right)
$$

where $s q r t M$ is the matrix square root function, see Appendix B.

According to the MCX method, the statistical estimates and its derivatives are located in the first column of $\left[P_{f}\left(\left[\theta_{i}\right]\right)\right],\left[\mu_{Z}\left(\left[\theta_{i}\right]\right)\right]$, and $\left[V_{Z}\left(\left[\theta_{i}\right]\right)\right]$. Let $\left[\theta_{i}\right]$ represent the matrix form of $\theta_{i}+h\left(i_{1}+i_{2}\right)$ with $h$ being the step size. The first column of the matrixvalued $\mathrm{POF},\left[P_{f}\left(\left[\theta_{i}\right]\right)\right]_{:, 1}$, is of the form 


$$
\left[P_{f}\left(\left[\theta_{i}\right]\right)\right]_{:, 1} \approx\left\{\begin{array}{c}
P_{f} \\
h \frac{\partial P_{f}}{\partial \theta_{i}} \\
h \frac{\partial P_{f}}{\partial \theta_{i}} \\
h^{2} \frac{\partial^{2} P_{f}}{\partial \theta_{i}^{2}}
\end{array}\right\}
$$

where \{\} denotes a column vector. Likewise, a response moment and its derivatives are determined by replacing $P_{f}$ in Eq. (27) with either $\mu_{Z}, V_{Z}$, or $\sigma_{z}$. Note that unlike the traditional SF method, the statistical estimates and its derivatives are obtained from a single multicomplex sampling-based simulation.

As an example, a second-order derivative of the POF with respect to $\theta_{i}$ requires a bicomplex number of the form $\theta_{i}+h\left(i_{1}+i_{2}\right)$. This bicomplex number is isomorphic to

$$
\left[\theta_{i}\right]=\left[\begin{array}{cccc}
\theta_{i} & -h & -h & 0 \\
h & \theta_{i} & 0 & -h \\
h & 0 & \theta_{i} & -h \\
0 & h & h & \theta_{i}
\end{array}\right]
$$

The POF and its derivatives are obtained from applying Eq. (27) as follows:

$$
\begin{aligned}
& P_{f} \approx\left[P_{f}\left(\left[\theta_{i}\right]\right)\right]_{1,1} \\
& \frac{\partial P_{f}}{\partial \theta_{i}} \approx \frac{\left[P_{f}\left(\left[\theta_{i}\right]\right)\right]_{2,1}}{h}=\frac{\left[P_{f}\left(\left[\theta_{i}\right]\right)\right]_{3,1}}{h} \\
& \frac{\partial^{2} P_{f}}{\partial \theta_{i}^{2}} \approx \frac{\left[P_{f}\left(\left[\theta_{i}\right]\right)\right]_{4,1}}{h^{2}}
\end{aligned}
$$

The second-order mixed partial derivatives are divided into two categories: intraderivatives and inter-derivatives. Intra-derivatives are those that are differentiated with 
respect to the PDF parameters of the same random variable, e.g., the mean and standard deviation of a normal distribution, $\partial^{2} P_{f} / \partial \mu_{1} \partial \sigma_{1}$, while inter-derivatives are differentiated with respect to independent variables' PDF parameters, e.g., the mean of a normal distribution and location of a lognormal distribution, $\partial^{2} P_{f} / \partial \mu_{1} \partial \lambda_{2}$.

Second-order intra-derivatives are determined as follows: Let $\theta_{i}^{1}$ and $\theta_{i}^{2}$ represent two parameters belonging to the same $\operatorname{PDF} f_{X_{i}}\left(\theta_{i}^{1}, \theta_{i}^{2}\right)$. The parameter $\theta_{i}^{1}$ is perturbed along the $i_{1}$ direction and $\theta_{i}^{2}$ is perturbed along the $i_{2}$ direction. Their multicomplex matrix representations are given as

$$
\begin{aligned}
& {\left[\theta_{i}^{1}\right]=\left[\begin{array}{cccc}
\theta_{i}^{1} & -h & 0 & 0 \\
h & \theta_{i}^{1} & 0 & 0 \\
0 & 0 & \theta_{i}^{1} & -h \\
0 & 0 & h & \theta_{i}^{1}
\end{array}\right]} \\
& {\left[\theta_{i}^{2}\right]=\left[\begin{array}{cccc}
\theta_{i}^{2} & 0 & -h & 0 \\
0 & \theta_{i}^{2} & 0 & -h \\
h & 0 & \theta_{i}^{2} & 0 \\
0 & h & 0 & \theta_{i}^{2}
\end{array}\right]}
\end{aligned}
$$

The multicomplex POF and multicomplex response moments are written as

$$
\begin{aligned}
& {\left[P_{f}\left(\left[\theta_{i}^{1}\right],\left[\theta_{i}^{2}\right]\right)\right] \approx \frac{1}{N} \sum_{k=1}^{N} I\left(\mathbf{x}_{k}\right) \cdot \frac{\left[f_{X_{i}}\left(x_{i, k} ;\left[\theta_{i}^{1}\right],\left[\theta_{i}^{2}\right]\right)\right]}{f_{X_{i}}}} \\
& {\left[\mu_{Z}\left(\left[\theta_{i}^{1}\right],\left[\theta_{i}^{2}\right]\right)\right] \approx \frac{1}{N} \sum_{k=1}^{N} z\left(\mathbf{x}_{k}\right) \cdot \frac{\left[f_{X_{i}}\left(x_{i, k} ;\left[\theta_{i}^{1}\right],\left[\theta_{i}^{2}\right]\right)\right]}{f_{X_{i}}}} \\
& {\left[V_{Z}\left(\left[\theta_{i}^{1}\right],\left[\theta_{i}^{2}\right]\right)\right] \approx \frac{1}{N} \sum_{k=1}^{N} z\left(\mathbf{x}_{k}\right)^{2} \cdot \frac{\left[f_{X_{i}}\left(x_{i, k} ;\left[\theta_{i}^{1}\right],\left[\theta_{i}^{2}\right]\right)\right]}{f_{X_{i}}}-\left[\mu_{Z}\left(\left[\theta_{i}^{1}\right],\left[\theta_{i}^{2}\right]\right)\right]^{2}}
\end{aligned}
$$


where $f_{X_{i}}=\left[f_{X_{i}}\left(x_{i, k} ;\left[\theta_{i}^{1}\right],\left[\theta_{i}^{2}\right]\right)\right]_{1,1}$.

Second-order inter-derivatives are calculated as follows: Let the PDF $f_{X_{i}}\left(\theta_{i}^{1}\right)$ be a function of $\theta_{i}^{1}$ and $f_{X_{j}}\left(\theta_{j}^{1}\right)$ be a function of $\theta_{j}^{1}$. The parameter $\theta_{i}^{1}$ is perturbed along the $i_{1}$ direction (Eq. (32)) and $\theta_{j}^{1}$ is perturbed along the $i_{2}$ direction. Substituting $\theta_{i}^{2}$ in Eq. (33) with $\theta_{j}^{1}$ gives the multicomplex matrix representation for $\left[\theta_{j}^{1}\right]$. The multicomplex POF and multicomplex response moments and their derivatives are calculated as follows:

$$
\begin{gathered}
{\left[P_{f}\left(\left[\theta_{i}^{1}\right],\left[\theta_{j}^{1}\right]\right)\right] \approx \frac{1}{N} \sum_{k=1}^{N} I\left(\mathbf{x}_{k}\right) \cdot \frac{\left[f_{X_{i}}\left(x_{i, k} ;\left[\theta_{i}^{1}\right]\right)\right]}{f_{X_{i}}} \frac{\left[f_{X_{j}}\left(x_{j, k} ;\left[\theta_{j}^{1}\right]\right)\right]}{f_{X_{j}}}} \\
{\left[\mu_{Z}\left(\left[\theta_{i}^{1}\right],\left[\theta_{j}^{1}\right]\right)\right] \approx \frac{1}{N} \sum_{k=1}^{N} z\left(\mathbf{x}_{k}\right) \cdot \frac{\left[f_{X_{i}}\left(x_{i, k} ;\left[\theta_{i}^{1}\right]\right)\right]}{f_{X_{i}}} \frac{\left[f_{X_{j}}\left(x_{j, k} ;\left[\theta_{j}^{1}\right]\right)\right]}{f_{X_{j}}}} \\
{\left[V_{Z}\left(\left[\theta_{i}^{1}\right],\left[\theta_{j}^{1}\right]\right)\right] \approx \frac{1}{N} \sum_{k=1}^{N} z\left(\mathbf{x}_{k}\right)^{2} \cdot \frac{\left[f_{X_{i}}\left(x_{i, k} ;\left[\theta_{i}^{1}\right]\right)\right]}{f_{X_{i}}} \frac{\left[f_{X_{j}}\left(x_{j, k} ;\left[\theta_{j}^{1}\right]\right)\right]}{f_{X_{j}}}-\left[\mu_{Z}\left(\left[\theta_{i}^{1}\right],\left[\theta_{j}^{1}\right]\right)\right]^{2}}
\end{gathered}
$$

where $f_{X_{i}}=\left[f_{X_{i}}\left(x_{i, k} ;\left[\theta_{i}^{1}\right]\right)\right]_{1,1}$ and $f_{X_{j}}=\left[f_{X_{j}}\left(x_{j, k} ;\left[\theta_{j}^{1}\right]\right)\right]_{1,1}$.

The POF and its derivatives are obtained from the following equations:

$$
\begin{aligned}
& P_{f} \approx\left[P_{f}\left(\left[\theta_{i}^{1}\right],\left[\theta_{j}^{1}\right]\right)\right]_{1,1} \\
& \frac{\partial P_{f}}{\partial \theta_{i}^{1}} \approx \frac{\left[P_{f}\left(\left[\theta_{i}^{1}\right],\left[\theta_{j}^{1}\right]\right)\right]_{2,1}}{h} \\
& \frac{\partial P_{f}}{\partial \theta_{j}^{1}} \approx \frac{\left[P_{f}\left(\left[\theta_{i}^{1}\right],\left[\theta_{j}^{1}\right]\right)\right]_{3,1}}{h} \\
& \frac{\partial^{2} P_{f}}{\partial \theta_{i}^{1} \partial \theta_{j}^{1}} \approx \frac{\left[P_{f}\left(\left[\theta_{i}^{1}\right],\left[\theta_{j}^{1}\right]\right)\right]_{4,1}}{h^{2}}
\end{aligned}
$$


The derivatives of the response moments can be obtained similarly by substituting the multicomplex POF in Eqs. (41-43) with a multicomplex response moment.

\subsection{Implementation for Various Random Variable Distributions}

The implementation of multicomplex mathematics to compute derivatives requires functions of matrices. For example, the multicomplex mathematics to compute the kernel function for a second-order derivative, $\left(\partial^{2} f_{X}(x, \boldsymbol{\theta}) / \partial \theta_{i}^{2}\right) / f(x, \boldsymbol{\theta})$, requires a bicomplex matrix representation for the parameter $\theta_{i}$. Therefore, the mathematical evaluation of the PDF must be modified to accept matrix input for the parameters. This process is described below for several probability distributions.

For convenience, the PDFs in this section are solely formulated in terms of the matrix-valued parameters. A perturbed parameter is equal to its corresponding matrix containing imaginary perturbations, and for an unperturbed parameter, the matrix is equal to the parameter value times the identity matrix, e.g., $[\mu]=\mu I$. Note that since the MCXSF method uses real samples, the sample $x_{i, k}$ must also be multiplied times the identity matrix before being used to evaluate the multicomplex PDF.

Non-normal distributions can be defined by either natural parameters or moments. If the moments, $\mu$ and $\sigma$, are perturbed then the appropriate multicomplex natural parameters must be obtained to evaluate the PDF. The relationship between moments and natural parameters for various distribution types are given in the following sections.

A step-by-step outline of the MCX-SF method is given below.

1. Perturb parameter(s) along imaginary unit direction(s).

2. Generate a real-valued random sample, $\mathbf{x}_{k}$. 
3. Evaluate the response function, $z\left(\mathbf{x}_{k}\right)$.

4. Evaluate the multicomplex PDFs, e.g., $\left[f_{X_{i}}\left(x_{i, k} I ;\left[\theta_{i}\right]\right)\right]$.

5. Calculate the multicomplex statistical estimates - Eqs. (23-26), Eqs. (34-36), Eqs. (37-39).

6. Extract the POF and the response moments and their derivatives from the first column of the matrix-valued estimates, see Eqs. (29-31) or Eqs. (40-43).

\subsubsection{Normal Distribution}

The PDF for the univariate normal distribution is written as

$$
f_{X}(x)=\frac{1}{\sigma \sqrt{2 \pi}} \exp \left[-\frac{(x-\mu)^{2}}{2 \sigma^{2}}\right]
$$

Its matrix equivalent is

$$
\left[f_{X}(x)\right]=\frac{I}{[\sigma] \sqrt{2 \pi}} \exp M\left[-\frac{(x I-[\mu])^{2}}{2[\sigma]^{2}}\right]
$$

where expM is the matrix exponential, see Appendix B.

\subsubsection{Bivariate Normal Distribution}

The PDF of the bivariate normal distribution is defined as

$$
\begin{gathered}
f_{X_{1}, X_{2}}\left(x_{1}, x_{2}\right)=\frac{1}{2 \pi \sigma_{x_{1}} \sigma_{x_{2}} \sqrt{1-\rho^{2}}} \exp \left(-\frac{1}{2\left(1-\rho^{2}\right)}\left[\frac{\left(x_{1}-\mu_{x_{1}}\right)^{2}}{\sigma_{x_{1}}^{2}}+\frac{\left(x_{2}-\mu_{x_{2}}\right)^{2}}{\sigma_{x_{2}}^{2}}-\right.\right. \\
\left.\left.2 \rho \frac{\left(x_{1}-\mu_{x_{1}}\right)\left(x_{2}-\mu_{x_{2}}\right)}{\sigma_{x_{1}} \sigma_{x_{2}}}\right]\right)
\end{gathered}
$$

where $\rho$ is the correlation coefficient. Its matrix extension is 


$$
\begin{array}{r}
{\left[f_{X_{1}, X_{2}}\left(x_{1}, x_{2}\right)\right]=\frac{I}{2 \pi\left[\sigma_{x_{1}}\right]\left[\sigma_{x_{2}}\right] \cdot \operatorname{sqrtM}\left(I-[\rho]^{2}\right)} \operatorname{expM}\left(-\frac{I}{2\left(I-[\rho]^{2}\right)}\left[\frac{\left(x_{1} I-\left[\mu_{x_{1}}\right]\right)^{2}}{\left[\sigma_{x_{1}}\right]^{2}}+\right.\right.} \\
\left.\left.\frac{\left(x_{2} I-\left[\mu_{x_{2}}\right]\right)^{2}}{\left[\sigma_{x_{2}}\right]^{2}}-2[\rho] \frac{\left(x_{1} I-\left[\mu_{x_{1}}\right]\right)\left(x_{2} I-\left[\mu_{x_{2}}\right]\right)}{\left[\sigma_{x_{1}}\right]\left[\sigma_{x_{2}}\right]}\right)\right]
\end{array}
$$

\subsubsection{Lognormal Distribution}

The PDF of a lognormal distribution is

$$
f_{X}(x)=\frac{1}{x \zeta \sqrt{2 \pi}} \exp \left[-\frac{(\ln (x)-\lambda)^{2}}{2 \xi^{2}}\right]
$$

where $\lambda$ and $\zeta$ are the location and scale parameter (natural parameters) of $\log \mathrm{x}$, respectively. Its matrix extension is written as

$$
\left[f_{X}(x)\right]=\frac{I}{x[\xi] \sqrt{2 \pi}} \exp M\left[-\frac{(\ln (x) I-[\lambda])^{2}}{2[\xi]^{2}}\right]
$$

Given the matrix form of the mean and standard deviation, the two natural parameters of the lognormal distribution can be computed as

$$
\begin{aligned}
& {[\lambda]=\log M\left([\mu]^{2} / \operatorname{sqrtM}\left([\sigma]^{2}+[\mu]^{2}\right)\right)} \\
& {[\zeta]=\operatorname{sqrtM}\left(\log M\left([\sigma]^{2} /[\mu]^{2}+I\right)\right)}
\end{aligned}
$$

where $\log M$ is the matrix logarithm, see Appendix B.

\subsubsection{Extreme Value Distrbution (Type I)}

The PDF of an extreme value distribution (type I) is

$$
f_{X}(x)=\frac{1}{b} \exp \left[-\frac{(x-a)}{b}\right] \cdot \exp \left[-\exp \left[-\frac{(x-a)}{b}\right]\right]
$$

where $a$ and $b$ are the location and scale parameter (natural parameters), respectively. The matrix extension of the PDF is 


$$
\left[f_{X}(x)\right]=\frac{I}{[b]} \exp M\left[-\frac{(x I-[a])}{[b]}\right] \cdot \exp M\left[-\exp M\left[-\frac{(x I-[a])}{[b]}\right]\right]
$$

The natural parameters, expressed in terms of the mean and standard deviation (moments), are

$$
\begin{gathered}
{[b]=\frac{[\sigma] \sqrt{6}}{\pi}} \\
{[a]=[\mu]-[b] \cdot \gamma}
\end{gathered}
$$

where $\gamma \approx 0.57721$ is the Euler-Mascheroni constant.

\subsubsection{2-Parameter Weibull Distribution}

Another popular distribution is the 2-parameter Weibull distribution. The PDF is defined as

$$
f_{X}(x)=\frac{a}{b}\left(\frac{x}{b}\right)^{a-1} \exp \left[-\left(\frac{x}{b}\right)^{a}\right]
$$

where $a$ and $b$ are called the shape and scale parameter (natural parameters), respectively. Its matrix form is given by

$$
\left[f_{X}(x)\right]=\frac{[a]}{[b]}\left(\frac{x I}{[b]}\right)^{[a]-I} \exp M\left[-\left(\frac{x I}{[b]}\right)^{[a]}\right]
$$

As shown in the above equation there are two terms on the right hand side of the equal sign that require the evaluation of a matrix raised to a matrix power. Using the property $A=\exp M(\log M(A))$, which is valid if $A$ is a square matrix with eigenvalues that have nonnegative real parts, Eq. (57) can be rewritten as

$$
\left[f_{X}(x)\right]=\frac{[a]}{[b]}[H(x I)][M(x I)]
$$

where 


$$
\begin{gathered}
{[H(x I)]=\exp M[([a]-I) \cdot \log M(x I /[b])]} \\
{[M(x I)]=\exp M[\exp M[[a] \cdot \log M(-x I /[b])]]}
\end{gathered}
$$

The mapping from natural parameters to the moments are given as

$$
\begin{gathered}
{[\mu]=[b] \cdot \Gamma \mathrm{M}(I+I /[a])} \\
{[\sigma]^{2}=[b]^{2}\left[\Gamma \mathrm{M}(I+2 I /[a])-(\Gamma \mathrm{M}(I+I /[a]))^{2}\right]}
\end{gathered}
$$

where $\Gamma \mathrm{M}$ is matrix version of the gamma function, see Appendix B. The above relationships are not algebraically invertible, so a numerical method to map from moments to natural parameters is needed. For this, a matrix form of the Newton-Raphson iteration method is used.

The Newton-Raphson formula in matrix form is given as

$$
[a]^{r+1}=[a]^{r}-\frac{\left[L\left([a]^{r}\right)\right]}{\left[L^{\prime}\left([a]^{r}\right)\right]}
$$

where $[a]^{r}$ is the matrix shape parameter at iteration $r$ and $L^{\prime}$ is the derivative of a realvalued function $L(a)=0$ with respect to $a$. The algorithm stops when a metric, e.g., the absolute difference between the current $[a]_{1,1}^{r}$ and next iteration $[a]_{1,1}^{r+1}$, is below a userdefined tolerance. The function $\left[L\left([a]^{r}\right)\right]$ is obtained as follows

$$
\begin{gathered}
\frac{[\mu]^{2}+[\sigma]^{2}}{[\mu]^{2}}=\frac{\Gamma \mathrm{M}\left(\frac{2 I}{[a]}+I\right)}{\Gamma \mathrm{M}\left(\frac{I}{[a]}+I\right)^{2}} \\
{\left[L\left([a]^{r}\right)\right]=\frac{\Gamma \mathrm{M}\left(\frac{2 I}{[a]^{r}}+I\right)}{\Gamma \mathrm{M}\left(\frac{I}{[a]^{r}}+I\right)^{2}}-\frac{[\mu]^{2}+[\sigma]^{2}}{[\mu]^{2}}}
\end{gathered}
$$

The derivative, $\left[L^{\prime}\left([a]^{r}\right)\right]$, is given by 


$$
\left[L^{\prime}\left([a]^{r}\right)\right]=2 \Gamma \mathrm{M}\left(\frac{2 I}{[a]^{r}}+I\right) \frac{\psi M\left(\frac{I}{[a]^{r}}+I\right)-\psi M\left(\frac{2 I}{[a]^{r}}+I\right)}{\left([a]^{r}\right)^{2} \Gamma \mathrm{M}\left(\frac{I}{[a]^{r}}+I\right)^{2}}
$$

where $\psi M$ is the matrix version of the digamma function, see Appendix B. After determining the matrix shape parameter $[a]$, Eq. (61) is used to determine the multicomplex scale parameter $[b]$.

\subsection{Variance Estimates of the Derivatives}

The derivative estimates computed from a Monte Carlo simulation are themselves random variables. Thus, it is important to calculate the variance estimates and confidence bounds of the sensitivities. The variance estimates for all derivatives follow the equation as reported in [Millwater and Osborn, 2006]

$$
V\left[\frac{\partial^{n} P_{f}}{\partial \theta_{i}^{n}}\right]=\frac{1}{N}\left(\frac{1}{N} \sum_{k=1}^{N}\left(I(\mathbf{x}) \cdot \kappa_{\theta_{i}}^{(n}(x)\right)^{2}-\left(\frac{\partial^{n} P_{f}}{\partial \theta_{i}^{n}}\right)^{2}\right) .
$$

It has been verified empirically that the sensitivities follow a normal distribution. Hence, the $95 \%$ confidence bounds are computed as

$$
\frac{\partial^{n} P_{f}}{\partial \theta_{i}^{n}}-1.96 \sqrt{V\left[\frac{\partial^{n} P_{f}}{\partial \theta_{i}^{n}}\right]} \leq \frac{\partial^{n} P_{f}}{\partial \theta_{i}^{n}} \leq \frac{\partial^{n} P_{f}}{\partial \theta_{i}^{n}}+1.96 \sqrt{V\left[\frac{\partial^{n} P_{f}}{\partial \theta_{i}^{n}}\right]} .
$$

\section{Numerical Examples}

The MCX-SF method is verified with three numerical examples. The first example consists of a linear response function with two independent normal random variables. The second example differs from the first example in that the two random 
variables are now correlated. Lastly, the MCX-SF method is applied to a probabilistic fracture mechanics problem. In all three examples the step size value was equal to $h=10^{-20}$.

\subsection{Linear Response Function With Two Normal Random Variables}

Let $x$ denote a random variable that is normally distributed with mean $\mu$ and standard deviation $\sigma$ (i.e., $x \sim N[\mu, \sigma]$ ). In the MCX-SF formulation two separate multicomplex PDFs are needed to compute the first to fourth order kernel functions of both $\mu$ and $\sigma$. The numerical kernel functions for a standard normal random variable were first compared against the analytical solutions. The kernel functions of $\mu$ and $\sigma$ are given in Figure 1 and Figure 2 respectively. As illustrated in the figures, the numerical (multicomplex-step) kernels compare well with the analytical kernels, see Millwater (2009).

In this first example, the system response was defined by the linear function.

$$
z(\mathbf{x})=x_{1}-x_{2}
$$

where $x_{1}$ and $x_{2}$ were normally distributed random variables with $\mu_{1}=10, \sigma_{1}=5$, $\mu_{2}=3, \sigma_{2}=2$.

Failure was defined when $x_{1} \leq x_{2}$, or equivalently, when $x_{1}-x_{2} \leq 0$. The exact analytical solution to the probability-of-failure for this type of problem is well known (Ang \& Tang, 2006) and analytical solutions to the first, second, third, and fourth order derivatives of the probability-of-failure with respect to the parameters of the random variables can be obtained in a straightforward manner. These analytical solutions were used to verify the numerical solutions that were calculated using the MCX-SF method. 
In the MCX method, a fourth-order derivative requires a minimum of a quadcomplex analysis. Due to the inherent properties of the MCX method, all lower order derivatives, first to third order derivatives, were computed as a byproduct of a quadcomplex analysis as shown in Eq. (23).

The probability-of-failure and its derivatives with respect to $\mu_{1}, \sigma_{1}, \mu_{2}$, and $\sigma_{2}$ were computed using Eq. (23). Since there are four parameters, $\mu_{1}, \sigma_{1}, \mu_{2}$, and $\sigma_{2}$, four separate quadcomplex-normally distributed PDFs were evaluated using Eq. (23): $\left[f_{X_{1}}\left(x_{1, k} ;\left[\mu_{1}\right]\right)\right],\left[f_{X_{1}}\left(x_{1, k} ;\left[\sigma_{1}\right]\right)\right],\left[f_{X_{2}}\left(x_{2, k} ;\left[\mu_{2}\right]\right)\right]$, and $\left[f_{X_{2}}\left(x_{2, k} ;\left[\sigma_{2}\right]\right)\right]$. The matrix extension of the normal PDF is given by Eq. (45). The quadcomplex matrix corresponding to the perturbation of a parameter $\theta_{i}$ is shown in Appendix C.

The POF and its derivatives were extracted from the first column of the resulting matrix as follows:

$$
\begin{gathered}
P_{f} \approx\left[P_{f}\left(\left[\theta_{i}\right]\right)\right]_{1,1} \\
\frac{\partial P_{f}}{\partial \theta_{i}} \approx \frac{\left[P_{f}\left(\left[\theta_{i}\right]\right)\right]_{2,1}}{h}=\frac{\left[P_{f}\left(\left[\theta_{i}\right]\right)\right]_{3,1}}{h}=\frac{\left[P_{f}\left(\left[\theta_{i}\right]\right)\right]_{5,1}}{h}=\frac{\left[P_{f}\left(\left[\theta_{i}\right]\right)\right]_{9,1}}{h} \\
\frac{\partial^{2} P_{f}}{\partial \theta_{i}^{2}} \approx \frac{\left[P_{f}\left(\left[\theta_{i}\right]\right)\right]_{4,1}}{h^{2}}=\frac{\left[P_{f}\left(\left[\theta_{i}\right]\right)\right]_{6,1}}{h^{2}}=\frac{\left[P_{f}\left(\left[\theta_{i}\right]\right)\right]_{7,1}}{h^{2}}=\frac{\left[P_{f}\left(\left[\theta_{i}\right]\right)\right]_{10,1}}{h^{2}}=\frac{\left[P_{f}\left(\left[\theta_{i}\right]\right)\right]_{11,1}}{h^{2}}=\frac{\left[P_{f}\left(\left[\theta_{i}\right]\right)\right]_{13,1}}{h^{2}} \\
\frac{\partial^{3} P_{f}}{\partial \theta_{i}^{3}} \approx \frac{\left[P_{f}\left(\left[\theta_{i}\right]\right)\right]_{8,1}}{h^{3}}=\frac{\left[P_{f}\left(\left[\theta_{i}\right]\right)\right]_{12,1}}{h^{3}}=\frac{\left[P_{f}\left(\left[\theta_{i}\right]\right)\right]_{14,1}}{h^{3}}=\frac{\left[P_{f}\left(\left[\theta_{i}\right]\right)\right]_{15,1}}{h^{3}} \\
\frac{\partial^{4} P_{f}}{\partial \theta_{i}^{4}} \approx \frac{\left[P_{f}\left(\left[\theta_{i}\right]\right)\right]_{16,1}}{h^{4}}
\end{gathered}
$$

The POF and its derivatives were obtained simultaneously in a single Monte Carlo simulation. 
The ratio of the numerical (MCX-SF) solution divided by the analytical solution for each derivative is shown in Table 1. Second-order mixed derivatives of the POF with respect to the parameters of the random variables are given in Table 2.

To verify variance estimate shown in Eq. (67), the variances of the derivatives of $P_{f}$ with respect to $\mu_{1}$ were computed at $10^{4}, 10^{5}$, and $10^{6}$ samples. In Figure 3 the lines denote the variances developed empirically from 10,000 MC simulations and the dots denote the variances computed using Eq. (66) as calculated from a single Monte Carlo analysis. As illustrated, the results indicate that Eq. (67) can accurately quantify the variance of any order derivative computed using MC sampling.

\subsection{Linear Response Function With Bivariate Normal Random Variables}

For the second example the random variables were normally distributed and correlated with a correlation coefficient of $\rho=0.9$. The matrix version of the bivariate normal density function is given in Section 3.2.2. The ratio of the derivatives, numerical divided by analytical, of the probability-of-failure with respect to each input parameter are given in Table 3 and 4.

As shown in the Table 1 and 3, for a fixed number of samples, it is observed that the variance of the MCX-SF derivative increases as the derivative order increases. This behavior is illustrated in Figure 4, which is a plot of the normalized variances of the derivatives with respect to $\sigma_{2}$ versus the number of samples. The empirical variance of each derivative was calculated using one thousand reevaluations. In Figure 4, the uncorrelated and correlated cases correspond to the solid lines and dashed lines respectively. Notice that the variance for all derivatives converge at a rate of $1 / \mathrm{N}$.

\subsection{Higher-Order Single-Flight Probability of Failure Sensitivities}


The calculation of the single-flight probability of failure (SFPOF) of an aircraft structure is an important metric for determining the airworthiness of an aircraft. Structural failure is assumed to occur due to unstable fracture (i.e., when the POF equation is given as, $K_{I} \geq K_{C}$, where $K_{I}$ denotes the stress intensity factor and $K_{C}$ is the fracture toughness). An equivalent statement is that failure occurs when the residual strength, $\sigma_{R S}$, is less than or equal to the max stress per flight, $\sigma_{E V D}$. The residual strength is defined as

$$
\sigma_{R S}=\frac{K_{C}}{\beta\left(a\left(a_{0}, t\right)\right) \sqrt{\pi a\left(a_{0}, t\right)}}
$$

where $a$ is the crack size at time $t, a_{0}$ is the initial crack size, and $\beta$ is the geometry correction factor (Anderson, 2005).

The following example is given in Tuegel et al. (2013). Consider a through crack at a hole under remote tension as depicted in Figure 5. The problem consists of a plate of width $W$, which contains a circular hole of radius $R$. The plate is subjected to a remote tensile stress $\sigma=\sigma_{E V D}$. There exists a through crack $a$ located on the right edge of the hole. The maximum applied stress, initial crack size, and fracture toughness were treated as random variables. The POF is written as

$$
P_{f}=\int_{0}^{\infty} \int_{-\infty}^{\infty}\left(1-F_{E V D}\left(\sigma_{R S}\left(a\left(a_{i}, t\right), K_{C}\right)\right)\right) f_{K_{C}} f_{a_{i}} d a_{i} d K_{C}
$$

The parameters and statistics are given in Table 5.

A sensitivity analysis of $P_{f}$ with respect to several statistical parameters at $t=12,000$ was conducted using the MCX-SF method and compared with a semi-analytic method. The semi-analytic approach consisted of symbolically differentiating the integral 
expression of the probability-of-failure with respect to a parameter of interest in MATLAB, and then numerically integrating that derivative expression using the built-in function integral2. Table 6 shows the ratio of the MCX-SF results to the semi-analytic results. Table 7 contains the MCX-SF estimate and 95\% confidence intervals of each derivative computed using Eq. (67).

\section{Discussion and Conclusions}

The development of high-order kernel functions that are needed for the probabilistic sensitivity analysis can theoretically be generated analytically using computer algebra systems. However, the numerical method presented here has some significant advantages, primarily: simplicity, extensibility, and adaptability.

MCX-SF is simple in concept and programming once the needed matrix functions are in place. No additional effort is needed to consider any order derivative including mixed partials; the size of the perturbation matrix and the location of the perturbed step size within the matrix controls the derivatives to be computed. Therefore, the user need only program the matrix PDF function to access kernels of any order.

Moreover, the evaluation of derivatives for the response variance and standard deviation highlight the simplicity in that the number of terms does not increase as the derivative order increases, as compared to a traditional approach as shown in Eq. (20). The derivative rules (i.e., power rule, chain rule, etc.) are essentially performed in a numerical fashion using multicomplex numbers. This eliminates the need to compute the Jacobian, Hessian, or any transformations in order to compute higher-order derivatives with respect to the mean and standard deviation for non-normal distributions. 
In contrast, an analytical development requires a lengthy number of terms to be programmed correctly for each distribution under consideration, including mixed partial derivatives. For example, the bivariate normal contains 5 parameters $\left(\mu_{1}, \mu_{2}, \sigma_{1}, \sigma_{2}, \rho\right)$, hence 5 first-order kernel functions are needed, 15 second-order functions, 15 third-order functions, etc.

The MCX-SF method is easily extensible to any order and other distributions. Once the code framework has been established, one merely changes the size of the matrix to obtain the desired derivatives; no additional programming is needed. As discussed above, the size of the matrix that must be processed to compute $n$th order and lower kernel functions is $2^{n}$. In addition, the addition of new probability distributions merely requires the programming of matrix-ready PDFs. If the needed matrix functions are available, e.g., exponential, log, power, square root,, new distributions can be added easily.

The method described here is particularly attractive for developing first order kernel functions in that only complex mathematics is required. Since complex mathematics is implemented in every programming language, no special matrix forms of the PDF are required. One simply evaluates the PDF using a complex parameters.

In summary, the capability to compute high-order derivatives of the probabilityof-failure or response moments with respect to parameters of the input random variables is facilitated through the use of multicomplex mathematics applied with the SF method. The use of MCX provides an easily extensible method to compute derivatives of any order of the required kernel functions. The requirement is that the random variable PDFs be evaluated in terms of functions of matrices, rather than real numbers. For the 
distributions considered, this requires algorithms for the matrix versions of the exponential, logarithm, square root, gamma and digamma functions. The addition of new probability distributions merely requires the programming of matrix-ready PDFs.

\section{Appendices}

\section{Appendix A. First-Order Kernel Function}

Although in practice the kernel functions are evaluated numerically using the MCX method, the complex step method can be shown to asymptotically yield the firstorder kernel functions as $h \rightarrow 0$, thus confirming the accuracy of the method.

For example, consider a normal distribution whose density function is given by Eq. (44). The kernel function of $\mu$ can be computed as $\kappa_{\mu}(x)=d \ln \left(f_{X}(x)\right) / d \mu$. The logarithm of the PDF is given as

$$
\begin{aligned}
\ln \left(f_{X}(x)\right) & =\ln (1 / \sigma \sqrt{2 \pi})-(x-\mu)^{2} / 2 \sigma^{2} \\
& =\ln (1 / \sigma \sqrt{2 \pi})-\left(x^{2}-2 x \mu+\mu^{2}\right) / 2 \sigma^{2}
\end{aligned}
$$

Adding a perturbation $h$ to the imaginary part of $\mu$ gives

$$
\begin{aligned}
\ln \left(f_{X}(x, \mu+i h)\right) & =\ln (1 / \sigma \sqrt{2 \pi})-\left(x^{2}-2 x(\mu+i h)+(\mu+i h)^{2}\right) / 2 \sigma^{2} \\
& =\ln (1 / \sigma \sqrt{2 \pi})-\left(x^{2}-2 x \mu-2 x h i+\mu^{2}+2 \mu h i-h^{2}\right) / 2 \sigma^{2}
\end{aligned}
$$

The imaginary coefficient in Eq. (A.2) is

$$
\begin{aligned}
\operatorname{Im}\left[\ln \left(f_{X}(x, \mu+i h)\right)\right] & =-(-2 x h+2 \mu h) / 2 \sigma^{2} \\
& =(x-\mu) h / \sigma^{2}
\end{aligned}
$$

Thus the first-order kernel function of $\mu$ is given as

$$
\kappa_{\mu}(x)=\frac{d \ln (f(x))}{d \mu} \approx \frac{\operatorname{Im}[\ln (f(x, \mu+i h))]}{h}=\frac{(x-\mu)}{\sigma^{2}}
$$

\section{Appendix B. Functions of Matrices}


The implementation of MCX-SF requires functions of matrices. In addition, MCX-SF has specific numerical requirements as the off-diagonal perturbation, $h$, is a very small number relative to the diagonal terms.

MATLAB contains several built-in matrix functions; however, in a preliminary study it was observed that some of these functions did not compute accurate multicomplex-step derivative results. One commonality between these built-in matrix functions was that their algorithms were based on the Schur decomposition of a matrix, which breaks down for matrices with repeated eigenvalues. As stated above, the MCX method uses diagonally dominant matrices, which produce repeated eigenvalues. The only built-in matrix function that performed acceptably for MCX-SF was the exponential function expm. All other functions, matrix square root, logarithm, gamma, and digamma functions relevant for multicomplex-step differentiation were programmed as described below. These algorithms were obtained from Higham (2008).

\section{Matrix Square Root}

A square root of matrix $A$ with real elements is a solution to $X^{2}=A$. If the eigenvalues of $A$ have nonnegative real parts, then there exists a unique square root $X$ of A. This unique solution is called the principal square root of $A$ and is written as $X=A^{1 / 2}$. In the examples presented, the matrix square root was evaluated using the Denman and Beavers iteration method (Higham, 2008). The algorithm is given as

$$
\begin{aligned}
& X_{k+1}=\frac{1}{2}\left(X_{k}+Y_{k}^{-1}\right) \\
& Y_{k+1}=\frac{1}{2}\left(Y_{k}+X_{k}^{-1}\right)
\end{aligned}
$$

where the initial guesses are $X_{0}=A$ and $Y_{0}=I$. Theoretically, 


$$
\begin{aligned}
& \lim _{k \rightarrow \infty} X_{k}=\operatorname{sqrtM}(A) \\
& \lim _{k \rightarrow \infty} Y_{k}=[\operatorname{sqrtM}(A)]^{-1}
\end{aligned}
$$

but in practice the user must specify when to terminate the iteration process. In the numerical examples presented here, the iteration process was terminated when the absolute difference between the diagonal element at the current and next iteration was equal to $10^{-5}$.

Alternatively, the integral representation of $A^{1 / 2}$ is defined as (Higham, 2008)

$$
\operatorname{sqrtM}(A)=\frac{2}{\pi} A \int_{0}^{\infty}\left(t^{2} I+A\right)^{-1} d t
$$

where $I$ is the identity matrix.

\section{Matrix Exponential}

The scaling and squaring method that is based upon Padé approximation was used to evaluate the exponential of a matrix (Moler and Van Loan, 2003). The built-in function expm, which uses the Padé approximation method, was used to calculate the matrix exponential because it produced accurate derivatives when used with MCX-SF.

\section{Matrix Logarithm}

A logarithm of matrix $A$ with real elements is a solution to $\operatorname{expm}(X)=A$. If the eigenvalues of $A$ have nonnegative real parts, there exists a principle logarithm $X$ of A. The principle logarithm is denoted by $\log M(A)$. In the numerical examples, the Gregory series was used to evaluate $\log M(A)$. The series is defined as

$$
\log M(\mathrm{~A})=-2 \sum_{k=0}^{\infty} \frac{1}{2 k+1}\left((I-A)(I+A)^{-1}\right)^{2 k+1}
$$


It is important to note that the series converges slowly when A is not close to I. In the examples, the series solution was terminated once the absolute difference between the diagonal element at the current and next iteration was equal to $10^{-5}$.

Alternatively, the integral representation of $\log M(A)$ is defined as (Higham, 2008)

$$
\log M(A)=\int_{0}^{1}(A-I)[t(A-I)+I]^{-1} d t
$$

\section{Matrix Gamma and Digamma Function}

Let $A$ be a matrix of real elements, which has eigenvalues that are greater than zero, and $\Gamma(A)$ represent the gamma function of $A$. The matrix gamma is defined as (Jódar \& Cortés, 1998)

$$
\Gamma \mathrm{M}(A)=\int_{0}^{\infty} \exp (-t) \cdot \operatorname{expm}[(A-I) \cdot \ln (t)] d t
$$

where exp and $l n$ are the exponential and logarithm, respectively. The digamma function, logarithmic derivative of the gamma function, of $A$ is denoted as $\psi M(A)$. The integral representation of the digamma is

$$
\psi M(A)=\int_{0}^{\infty}\left(\frac{\exp (-t)}{t} I-\frac{\operatorname{expm}[-A t]}{1-\exp (-t)}\right) d t
$$

\section{Appendix C. Quadcomplex Matrix}

A first, second, third, and fourth-order derivative is computed using the quadcomplex number $\theta_{i}+h\left(i_{1}+i_{2}+i_{3}+i_{4}\right)$, which is isomorphic to 


$$
\left[\theta_{i}\right]=\left[\begin{array}{cccccccccccccccc}
\theta_{i} & -h & -h & 0 & -h & 0 & 0 & -h & 0 & 0 & 0 & 0 & 0 & 0 & 0 & 0 \\
h & \theta_{i} & 0 & -h & 0 & -h & 0 & 0 & -h & 0 & 0 & 0 & 0 & 0 & 0 & 0 \\
h & 0 & \theta_{i} & -h & 0 & 0 & -h & 0 & 0 & -h & 0 & 0 & 0 & 0 & 0 & 0 \\
0 & h & h & \theta_{i} & 0 & 0 & 0 & -h & 0 & 0 & -h & 0 & 0 & 0 & 0 & 0 \\
0 & 0 & 0 & 0 & \theta_{i} & -h & -h & 0 & 0 & 0 & 0 & -h & 0 & 0 & 0 & 0 \\
h & 0 & 0 & 0 & h & \theta_{i} & 0 & -h & 0 & 0 & 0 & 0 & -h & 0 & 0 & 0 \\
0 & h & 0 & 0 & h & 0 & \theta_{i} & -h & 0 & 0 & 0 & 0 & 0 & -h & 0 & 0 \\
0 & 0 & h & 0 & 0 & h & h & \theta_{i} & 0 & 0 & 0 & 0 & 0 & 0 & -h & 0 \\
h & 0 & 0 & h & 0 & 0 & 0 & 0 & \theta_{i} & -h & -h & 0 & -h & 0 & 0 & -h \\
0 & h & 0 & 0 & 0 & 0 & 0 & 0 & h & \theta_{i} & 0 & -h & 0 & -h & 0 & 0 \\
0 & 0 & h & 0 & 0 & 0 & 0 & 0 & h & 0 & \theta_{i} & -h & 0 & 0 & -h & 0 \\
0 & 0 & 0 & h & 0 & 0 & 0 & 0 & 0 & h & h & \theta_{i} & 0 & 0 & 0 & -h \\
0 & 0 & 0 & 0 & h & 0 & 0 & h & 0 & 0 & 0 & 0 & \theta_{i} & -h & -h & 0 \\
0 & 0 & 0 & 0 & 0 & h & 0 & 0 & h & 0 & 0 & 0 & h & \theta_{i} & 0 & -h \\
0 & 0 & 0 & 0 & 0 & 0 & h & 0 & 0 & h & 0 & 0 & h & 0 & \theta_{i} & -h \\
0 & 0 & 0 & 0 & 0 & 0 & 0 & h & 0 & 0 & h & 0 & 0 & h & h & \theta_{i}
\end{array}\right]
$$

\section{Acknowledgements}

This research was sponsored by the Air Force Office of Scientific Research (AFOSR)

grant W911NF-11-1-0208 and the Office of Naval Research N00014-14-1-0004.

\section{References}

Anderson, T.L. (2005). Fracture Mechanics: Fundamentals and Applications, Third Edition: Taylor \& Francis.

Ang, A.H.S., \& Tang, W.H. (2007). Probability Concepts in Engineering: Emphasis on Applications to Civil and Environmental Engineering: Wiley.

Arnst, M., \& Ponthot, J.-P.. (2014). An Overview of Nonintrusive Characterization, Propagation, and Sensitivity Analysis of Uncertainty in Computational Mechanics International Journal for Uncertainty Quantification, 4(5), 387-421. doi: 10.1615/Int.J.UncertaintyQuantification.2014006990

Garza, J., \& Millwater, H. (2012). Sensitivity of probability-of-failure estimates with respect to probability of detection curve parameters. International Journal of Pressure Vessels and Piping, 92(0), 84-95. doi: http://dx.doi.org/10.1016/j.ijpvp.2011.11.009 
Higham, N.J. (2008). Functions of Matrices: Theory and Computation: Society for Industrial and Applied Mathematics.

Jódar, L., \& Cortés, J. C. (1998). Some properties of Gamma and Beta matrix functions. Applied Mathematics Letters, 11(1), 89-93. doi: http://dx.doi.org/10.1016/S08939659(97)00139-0

Kleijnen, Jack, \& Rubinstein, Reuven Y. (1996). Optimization and sensitivity analysis of computer simulation models by the score function method. European Journal of Operational Research 88, 413-427.

Lantoine, Gregory, Russell, Ryan P., \& Dargent, Thierry. (2012). Using Multicomplex Variables for Automatic Computation of High-Order Derivatives. ACM Trans. Math. Softw., 38(3), 1-21. doi: 10.1145/2168773.2168774

Lee, I., Choi, K. K., Noh, Y., Zhao, L., \& Gorsich, D. (2011). Sampling-Based Stochastic Sensitivity Analysis Using Score Functions for RBDO Problems With Correlated Random Variables. Journal of Mechanical Design, 133(2), 021003-021003. doi: $10.1115 / 1.4003186$

MATLAB version 7.14.0.739. Natick, Massachusetts: The MathWorks Inc., 2012.

Millwater, H. (2009). Universal properties of kernel functions for probabilistic sensitivity analysis. Probabilistic Engineering Mechanics, 24(1), 89-99. doi: http://dx.doi.org/10.1016/j.probengmech.2008.01.005

Millwater, H., \& Osborn, R.W. (2006). Probabilistic Sensitivities for Fatigue Analysis of Turbine Engine Disks. International Journal of Rotating Machinery, 2006, 12. doi: $10.1155 / \mathrm{ijrm} / 2006 / 28487$

Millwater, H., Singh, G., \& Cortina, M. (2012). Development of a localized probabilistic sensitivity method to determine random variable regional importance. Reliability Engineering \& System Safety, 107(0), 3-15. doi: http://dx.doi.org/10.1016/j.ress.2011.04.003

Millwater, H., Bates, A., \& Vazquez, E. (2011). Probabilistic Sensitivity Methods for Correlated Normal Variables. International Journal of Reliability and Safety, 5(1), 1-20.

Millwater, H., \& Feng, Y. (2011). Probabilistic Sensitivity Analysis with respect to Bounds of Truncated Random Variables. ASME J. Mech. Des. 133(6), doi: $10.1115 / 1.4003819$

Moler, C., \& Van Loan, C. (2003). Nineteen Dubious Ways to Compute the Exponential of a Matrix, Twenty-Five Years Later. SIAM Review, 45(1), 3-49. doi: $10.1137 / \mathrm{S} 00361445024180$ 
Price, G. Baley. (1991). An Introduction to Multicomplex Spaces and Functions.

Rahman, S. (2009). Stochastic sensitivity analysis by dimensional decomposition and score functions. Probabilistic Engineering Mechanics, 24(3), 278-287. doi: http://dx.doi.org/10.1016/j.probengmech.2008.07.004

Rubinstein, R.Y., (1986) The score function approach for sensitivity analysis of computer simulation models, Mathematics and Computers in Simulation, V 28, 5, pp. 351379

Rubinstein, R.Y. (1989) Sensitivity Analsis and Performance Extrapolation for computer Simulation Models, Operations Research, V 37, No. 1, pp 72-81

Rubinstein, R.Y. , \& Shapiro, A. (1993). Discrete event systems: Sensitivity analysis and stochastic optimization by the score function method: Wiley.

Rubinstein, R.Y. (1989) "Optimization of computer simulation models with rare events," European Journal of Operational Research, V 99, No. 1 pp 89-112

Saltelli, A., Ratto, M., Andres, T., Campolongo, F., Cariboni, J., Gatelli, D., Tarantola, S. (2008). Global Sensitivity Analysis: The Primer: Wiley.

Shirinkam, S., \& Millwater, H.R. (2014). Multicomplex Taylor Series Expansion for Computing High Order Derivatives. International Journal of Applied Mathematics, 27(4). doi:http://dx.doi.org/10.12732/ijam.v27i4.2

Sues, R.H., \& Cesare, M.A. (2005). System reliability and sensitivity factors via the MPPSS method. Probabilistic Engineering Mechanics, 20(2), 148-157. doi: http://dx.doi.org/10.1016/j.probengmech.2005.02.001

Tuegel, E.J., Bell, R.P., Berens, A.P., Brussat, T., Cardinal, J.W., Gallagher, J.P., \& Rudd, J. (2013). Aircraft Structural Reliability and Risk Analysis Handbook, Volume 1: Basic Analysis Methods, AFRL-RQ-WP-TR-2013-0132, http://www.dtic.mil/dtic/tr/fulltext/u2/a587824.pdf

Wang, P., Lu, Z., Hu, J., \& Zhou, C. (2014). Sensitivity analysis of the variance contributions with respect to the distribution parameters by the kernel function. Computers \& Mathematics with Applications, 67(10), 1756-1771. doi: http://dx.doi.org/10.1016/j.camwa.2014.04.007

$\mathrm{Wu}, \mathrm{Y}$. T. (1994). Computational methods for efficient structural reliability and reliability sensitivity analysis. AIAA Journal, 32(8), 1717-1723. doi: $10.2514 / 3.12164$ 
Wu, Y. T., \& Mohanty, Sitakanta. (2006). Variable screening and ranking using sampling-based sensitivity measures. Reliability Engineering \& System Safety, 91(6), 634-647. doi: http://dx.doi.org/10.1016/j.ress.2005.05.004 


\section{List of Figures}

Figure 1. Standard normal kernels of the mean parameter, $\boldsymbol{\kappa}_{\mu}^{(n)}$

Figure 2. Standard normal kernels of the standard deviation parameter, $\kappa_{\sigma}^{(n)}$

Figure 3. A comparison of the variances of the derivatives from a single Monte Carlo simulation to 10,000 derivative estimates.

Figure 4. Variances of higher-order probability-of-failure derivatives with respect to $\sigma_{2}$

Figure 5: Single Through Crack in a Plate Emanating From a Circular Hole 


\section{List of Tables}

Table 1: Comparison of derivatives computed numerically with analytical results, MCXSF divided by analytic. Linear response function with normal variables.

Table 2: Comparison of second-order mixed derivatives, MCX-SF divided by analytic. Linear response function with normal variables.

Table 3: Comparison of derivatives computed numerically with analytic results, MCX-SF divided by analytic. Linear response function with correlated normal variables.

Table 4: Comparison of second-order mixed derivatives, MCX-SF divided by analytic. Linear response function with correlated normal variables.

Table 5: Model parameters and statistics

Table 6: Comparison of derivatives computed numerical with analytic results, MCX-SF divided by semi-analytic. Fracture Mechanics Example.

Table 7: MCX-SF derivatives and confidence bounds. Fracture Mechanics Example. 


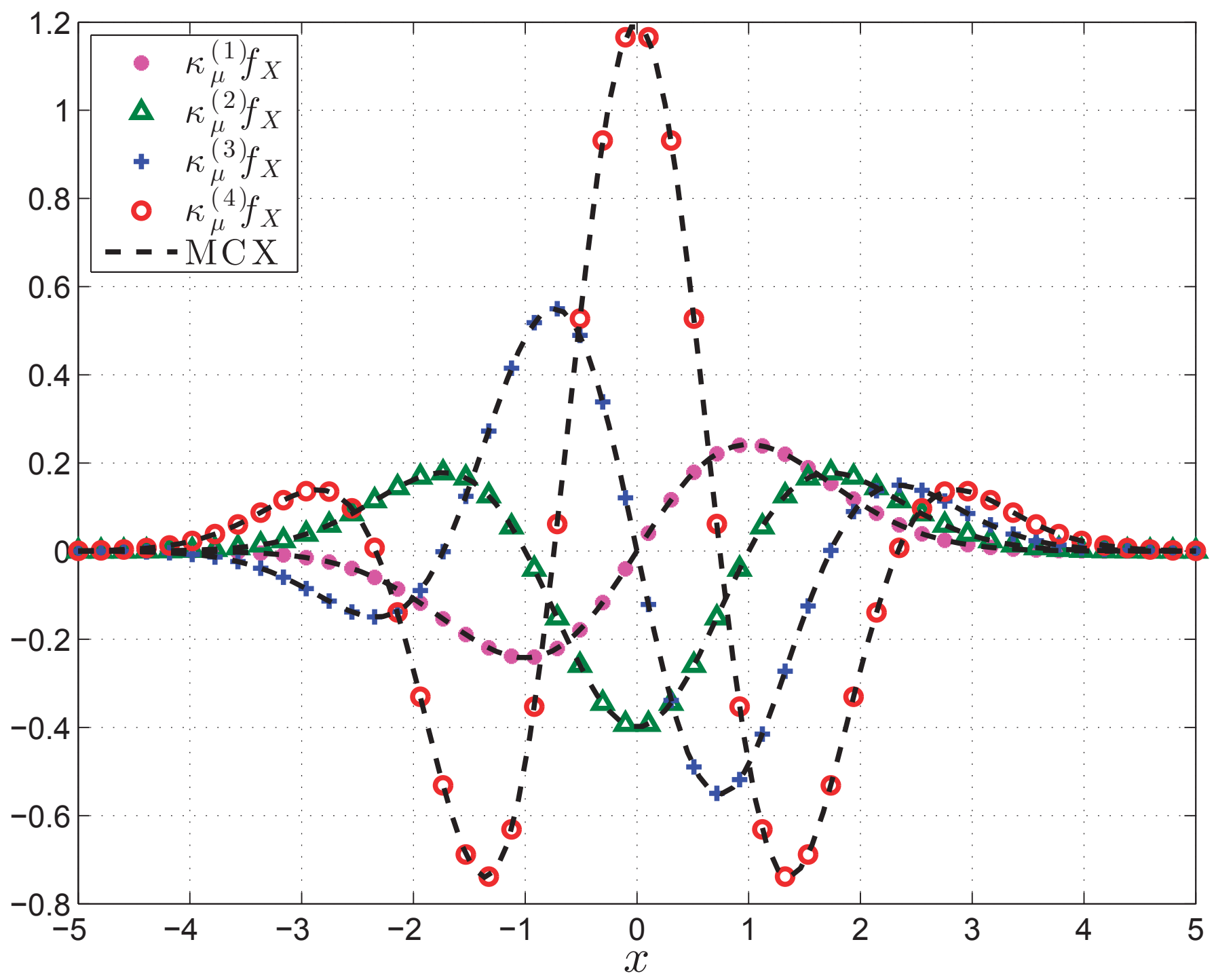




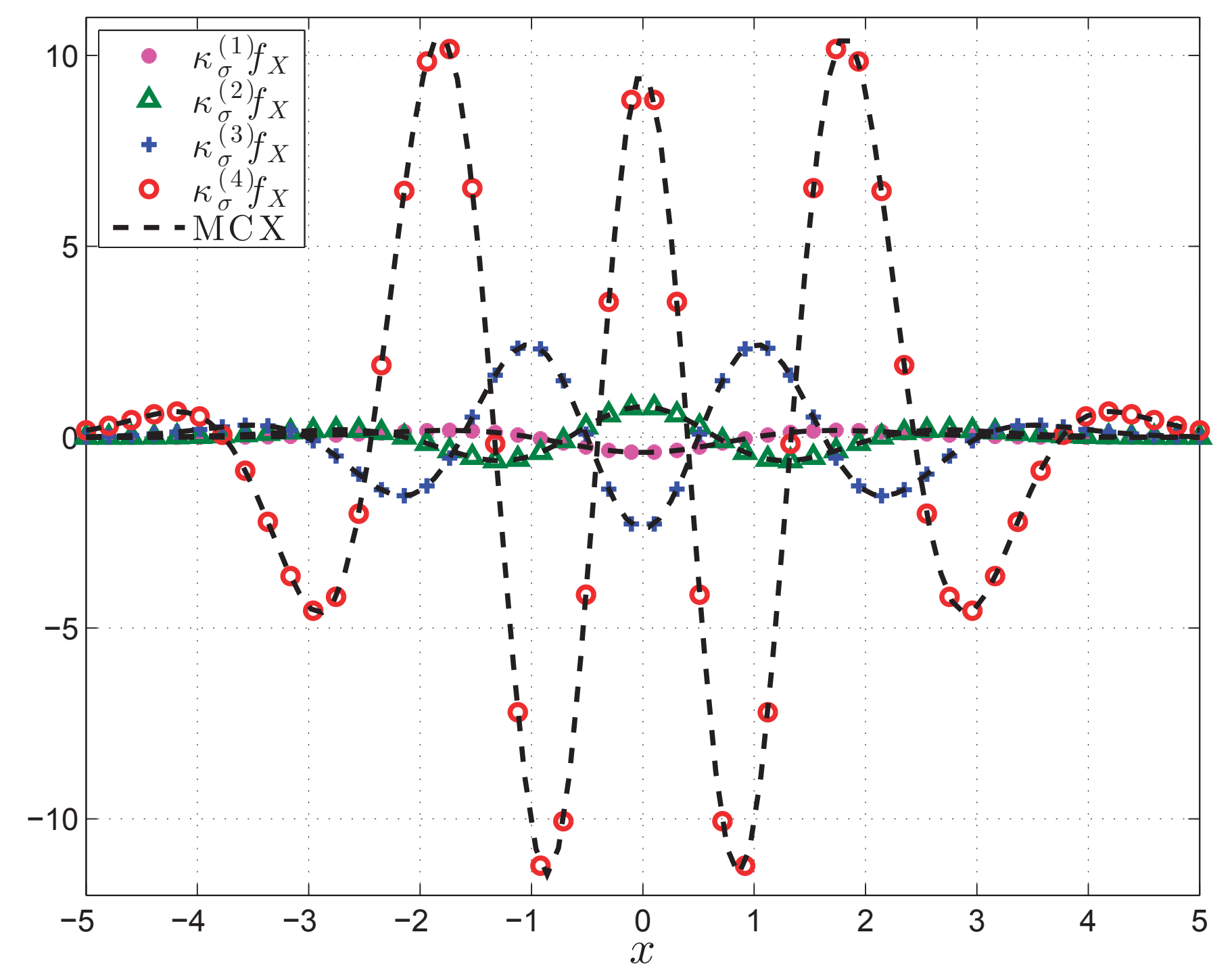

Figure2 


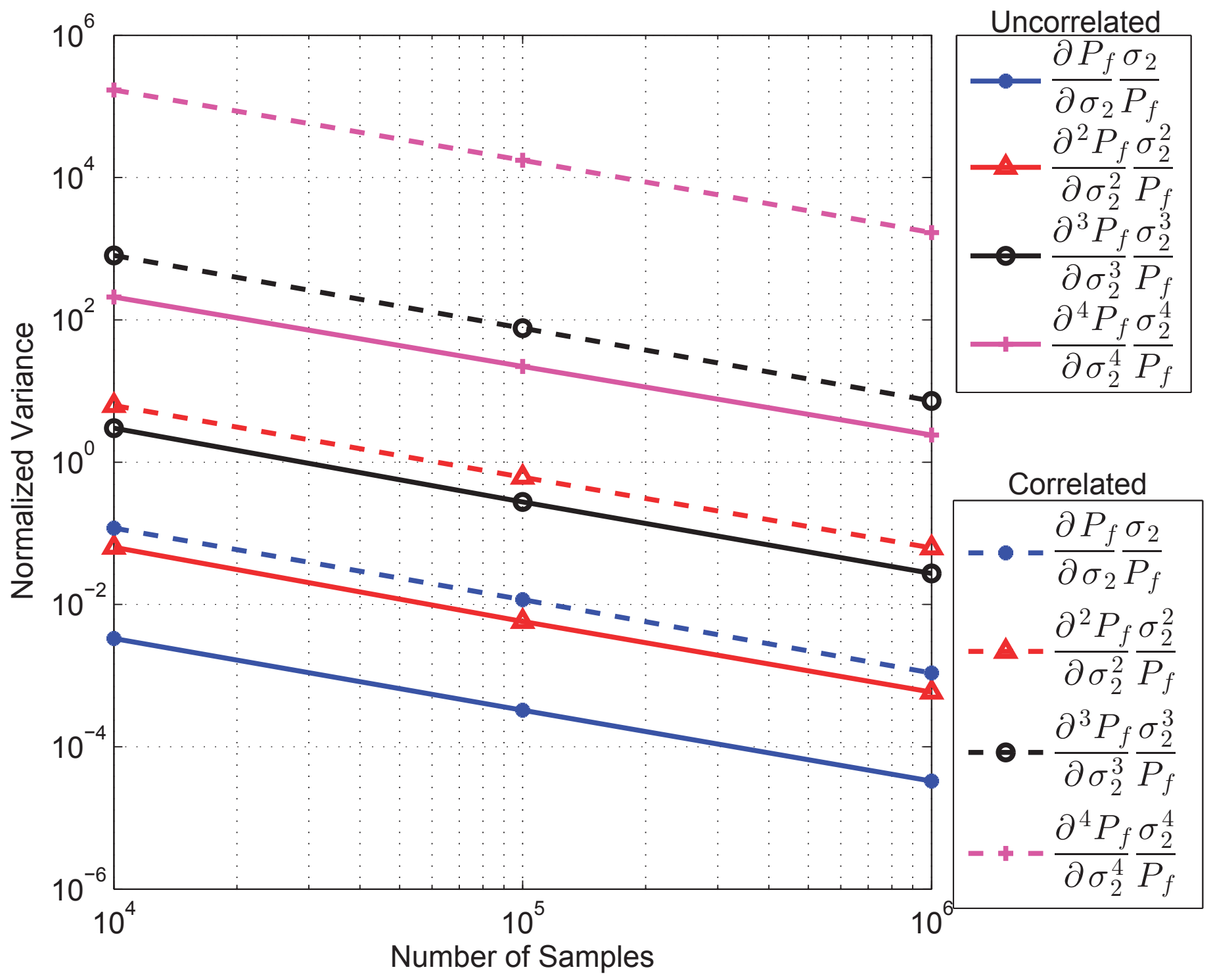




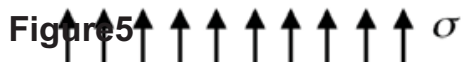

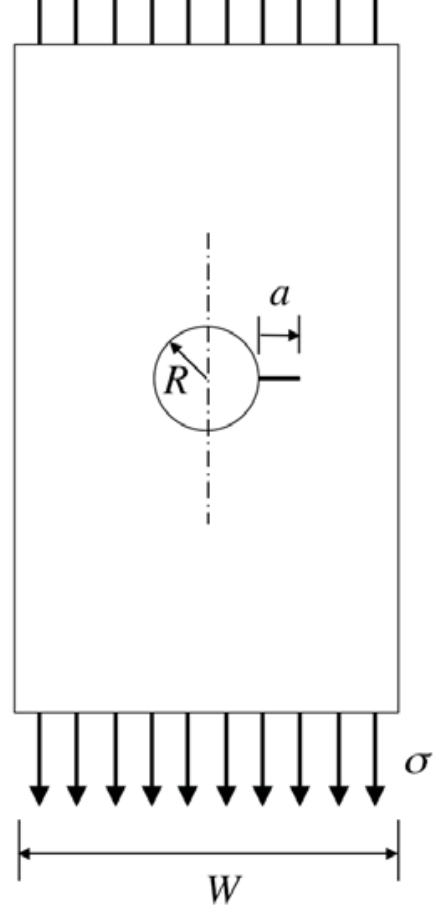


Table 1: Comparison of derivatives computed numerically with analytical results, MCXSF divided by analytic. Linear response function with normal variables.

\begin{tabular}{|c|c|c|c|c|}
\hline & \multicolumn{3}{|c|}{$\square_{i}$} \\
\cline { 2 - 5 } & $\square_{1}$ & $\square_{1}$ & $\square_{2}$ & $\square_{2}$ \\
\hline$\frac{\square P_{f}}{\square \square_{i}}$ & 1.000 & 1.000 & 1.000 & 1.000 \\
\hline$\frac{\square^{2} P_{f}}{\square{ }_{i}^{2}}$ & 1.000 & 1.000 & 1.000 & 1.000 \\
\hline$\frac{\square^{3} P_{f}}{\square_{i}^{3}}$ & 0.9997 & 0.9990 & 1.003 & 1.014 \\
\hline$\frac{\square^{4} P_{f}}{\square \sqrt{4}}$ & 0.9997 & 1.003 & 1.000 & 0.7464 \\
\hline
\end{tabular}

\footnotetext{
$* 10^{9}$ Monte Carlo samples
} 
Table 2: Comparison of second-order mixed derivatives, MCX-SF divided by analytic. Linear response function with normal variables.

\begin{tabular}{|c|c|c|c|c|c|}
\hline \multicolumn{2}{|c|}{$\frac{\square^{2} P_{f}}{\nabla_{i} \square_{j}}$} & \multicolumn{4}{|c|}{$\square_{j}$} \\
\cline { 2 - 6 } & $\square_{1}$ & 1.000 & 1.000 & $\square_{1}$ & $\square_{2}$ \\
\hline \multirow{4}{*}{$\square_{i}$} & $\square_{1}$ & - & 1.000 & 0.9998 & 0.9987 \\
\cline { 2 - 6 } & $\square_{2}$ & - & - & 1.000 & 1.000 \\
\cline { 2 - 6 } & $\square_{2}$ & - & - & - & 1.000 \\
\cline { 2 - 6 } & $\square_{2}$ & - & & & 1.000 \\
\hline
\end{tabular}

$* 10^{9}$ Monte Carlo samples 
Table 3: Comparison of derivatives computed numerically with analytic results, MCX-SF divided by analytic. Linear response function with correlated normal variables.

\begin{tabular}{|c|c|c|c|c|c|}
\hline & \multicolumn{5}{|c|}{$\square_{i}$} \\
\cline { 2 - 6 } & $\square_{1}$ & $\square_{1}$ & $\square_{2}$ & $\square_{2}$ & $\square$ \\
\hline$\frac{\square P_{f}}{\square \square_{i}}$ & 1.000 & 1.000 & 1.000 & 0.9995 & 1.000 \\
\hline$\frac{\square^{2} P_{f}}{\square \square_{i}^{2}}$ & 1.000 & 1.000 & 1.000 & 1.000 & 0.9990 \\
\hline$\frac{\square^{3} P_{f}}{\square{ }_{i}^{3}}$ & 1.000 & 1.000 & 1.000 & 0.9931 & 1.019 \\
\hline$\frac{\square^{4} P_{f}}{\square P_{i}^{4}}$ & 0.9995 & 1.003 & 0.9908 & 1.014 & 0.8469 \\
\hline
\end{tabular}

* $10^{9}$ Monte Carlo samples 
Table 4: Comparison of second-order mixed derivatives, MCX-SF divided by analytic. Linear response function with correlated normal variables.

\begin{tabular}{|c|c|c|c|c|c|c|}
\hline \multicolumn{2}{|c|}{$\frac{\square^{2} P_{f}}{\square \square_{i} \square_{j}}$} & \multicolumn{5}{|c|}{$\square_{i}$} \\
\cline { 2 - 7 } & $\square_{1}$ & 1.000 & 0.9997 & 1.000 & 1.000 & 0.9995 \\
\cline { 2 - 7 }$\square_{j}$ & $\square_{1}$ & - & 1.000 & 1.000 & 0.9997 & 0.9992 \\
\cline { 2 - 7 } & $\square_{2}$ & - & - & 1.000 & 1.000 & 1.000 \\
\cline { 2 - 7 } & $\square_{2}$ & - & - & - & 1.000 & 0.9972 \\
\cline { 2 - 7 } & $\square$ & - & - & - & - & 0.9990 \\
\hline
\end{tabular}

* $10^{9}$ Monte Carlo samples 
Table 5: Model parameters and statistics

\begin{tabular}{|c|c|}
\hline Variable & Description \\
\hline Radius (in.), $R$ & 0.125 in. \\
\hline Width (in.), $W$ & 1,000 in. (semi-infinite plate) \\
\hline Exponential growth parameter, $Q$ & 0.000293 \\
\hline Maximum applied stress (ksi), $\square_{E V D}$ & $\begin{array}{l}\text { Gumbel } \\
\text { Natural Parameters: } \\
\text { Location }=a=14.5 \\
\text { Scale }=b=0.800 \\
\text { Moments: } \\
\square_{\square_{E V D}}=14.96 \\
\square_{\square_{E V D}}=1.026\end{array}$ \\
\hline Initial crack size (in), $a_{0}$ & $\begin{array}{c}\quad \underline{\text { Lognormal }} \\
\text { Natural Parameters: } \\
\square=\square 6.420 \\
\square=1.113 \\
\text { Moments: } \\
\quad \square_{a_{0}}=3.025 \times 10^{\natural 3} \\
\square_{a_{0}}=4.737 \times 10^{\square}\end{array}$ \\
\hline Fracture Toughness ( $\mathrm{ksi} \sqrt{\mathrm{in}}), K_{C}$ & $\begin{array}{cl}\text { Moments: } & \text { Normal } \\
\square_{K_{C}}=34.80 & \\
\square_{K_{C}}=3.900 & \end{array}$ \\
\hline
\end{tabular}


Table 6: Comparison of derivatives computed numerical with semi-analytic results, MCX-SF divided by semi-analytic. Fracture Mechanics Example.

\begin{tabular}{|c|c|c|c|c|}
\hline & \multicolumn{3}{|c|}{$\square_{i}$} \\
\cline { 2 - 5 } & $\square_{a_{0}}$ & $\square_{a_{0}}$ & $\square_{K_{C}}$ & $\square_{K_{C}}$ \\
\hline$\frac{\square P_{f}(12,000)}{\square_{i}}$ & 0.9997 & 0.9989 & 0.9954 & 0.9930 \\
\hline$\frac{\square^{2} P_{f}(12,000)}{\square \square_{i}^{2}}$ & 1.008 & 1.001 & 0.9925 & 1.008 \\
\hline$\frac{\square^{3} P_{f}(12,000)}{\square P_{i}^{3}}$ & 0.9897 & 0.9924 & 0.9970 & 1.051 \\
\hline$\frac{\square^{4} P_{f}(12,000)}{\square r_{i}^{4}}$ & 0.9966 & 1.005 & 1.018 & 1.053 \\
\hline
\end{tabular}

* $10^{9}$ Monte Carlo samples 
Table 7: MCX-SF derivatives and confidence bounds. Fracture Mechanics Example.

\begin{tabular}{|c|c|c|c|c|}
\hline & \multicolumn{4}{|c|}{$\square_{i}$} \\
\hline & $\square_{a_{0}}$ & $\square_{a_{0}}$ & $\square_{K_{C}}$ & $\square_{K_{C}}$ \\
\hline$\square P_{f}(12,000)$ & $-9.156 \leq-9.108$ & $1.816 \leq 1.824 \leq$ & $\begin{array}{c}-4.425 \leq-4.399 \\
<-4374\end{array}$ & $4.663 \leq 4.714 \leq$ \\
\hline$\overline{\square \nabla_{i}}$ & $\left(\times 10^{-2}\right)$ & $\left(\mathrm{x} 10^{-1}\right)$ & $\begin{array}{c}=-4.3 / 4 \\
\left(\times 10^{-5}\right)\end{array}$ & $\left(\times 10^{-5}\right)$ \\
\hline$\square^{2} P_{f}(12,000)$ & $2.443 \leq 2.481 \leq$ & $8.762 \leq 8.819 \leq$ & $1.198 \leq 1.208 \leq$ & $3.161 \leq 3.256 \leq$ \\
\hline$\overline{\nabla_{i}^{2}}$ & $\begin{array}{l}2.519 \\
(\mathrm{x} 10)\end{array}$ & $\begin{array}{l}8.876 \\
(\mathrm{x} 10)\end{array}$ & $\begin{array}{l}1.221 \\
\left(\mathrm{x} 10^{-5}\right)\end{array}$ & $\begin{array}{l}3.350 \\
\left(\mathrm{x} 10^{-5}\right)\end{array}$ \\
\hline${ }^{3} P_{f}(12,000)$ & $3.254 \leq 3.297 \leq$ & $-3.712 \leq-3.681$ & $-3.843 \leq-3.763$ & $-2.834 \leq 3.121 \leq$ \\
\hline$\overline{\square \beta_{i}^{3}}$ & $\begin{array}{l}3.339 \\
\left(\times 10^{4}\right)\end{array}$ & $\begin{array}{c}\leq-3.649 \\
\left(x 10^{4}\right)\end{array}$ & $\begin{array}{c}\leq-3.684 \\
\left(\times 10^{-6}\right)\end{array}$ & $\begin{array}{l}3.408 \\
\left(\times 10^{-5}\right)\end{array}$ \\
\hline$\square^{4} P_{f}(12,000)$ & $-7.427 \leq-7.360$ & $-2.841 \leq-2.806$ & $1.289 \leq 1.346 \leq$ & $-3.470 \leq 4.566 \leq$ \\
\hline$\nabla_{i}^{4}$ & $\begin{array}{c}\leq-7.293 \\
\left(\mathrm{x} 10^{7}\right)\end{array}$ & $\begin{array}{c}\leq-2.771 \\
\left(\mathrm{x} 10^{7}\right)\end{array}$ & $\begin{array}{l}1.402 \\
\left(\times 10^{-6}\right)\end{array}$ & $\begin{array}{r}5.663 \\
\left(\times 10^{-5}\right)\end{array}$ \\
\hline
\end{tabular}

\footnotetext{
* $10^{9}$ Monte Carlo samples
} 